\title{
Weakly Supervised Object Localization with Multi-Fold Multiple Instance Learning
}

\author{
Ramazan Gokberk Cinbis, Jakob Verbeek, and Cordelia Schmid, Fellow, IEEE
}

\begin{abstract}
Object category localization is a challenging problem in computer vision. Standard supervised training requires bounding box annotations of object instances. This time-consuming annotation process is sidestepped in weakly supervised learning. In this case, the supervised information is restricted to binary labels that indicate the absence/presence of object instances in the image, without their locations. We follow a multiple-instance learning approach that iteratively trains the detector and infers the object locations in the positive training images. Our main contribution is a multi-fold multiple instance learning procedure, which prevents training from prematurely locking onto erroneous object locations. This procedure is particularly important when using high-dimensional representations, such as Fisher vectors and convolutional neural network features. We also propose a window refinement method, which improves the localization accuracy by incorporating an objectness prior. We present a detailed experimental evaluation using the PASCAL VOC 2007 dataset, which verifies the effectiveness of our approach.
\end{abstract}

Index Terms-Weakly supervised learning, object detection

\section{INTRODUCTION}

$\mathrm{O}$ VER the last decade significant progress has been made in object category localization, as witnessed by the PASCAL VOC challenges [20]. Training state-of-the-art object detectors, however, requires bounding box annotations of object instances, which are costly to acquire.

Weakly supervised learning (WSL) refers to methods that rely on training data with incomplete ground-truth information to learn recognition models. For object detection, WSL from image-wide labels that indicate the presence of instances of a category in images has recently been intensively studied as a way to remove the need for bounding box annotations, see e.g., [4], [8], [12], [15], [17], [35], [37], [38], [40], [43], [45], [46], [47], [53]. Such methods can potentially leverage the large amount of tagged images on the internet as a data source to train object detectors. We give an overview of the most relevant related work in Section 2.

Other examples of WSL include learning face recognition models from image captions [6], or subtitle and script information [19]. Yet another example is learning semantic segmentation models from image-wide category labels [51]. Most WSL approaches are based on latent variable models to account for the missing ground-truth information. Multiple instance learning (MIL) [18] handles cases where the weak supervision indicates that at least one positive instance is present in a set of examples. More advanced inference and learning methods are used in cases where the latent variable structure is more complex, see e.g., [17], [40], [51]. Besides

- R.G. Cinbis is with the Department of Computer Engineering, Bilkent University, Ankara,Turkey.E-mail: gcinbis@cs.bilkent.edu.tr.

- J. Verbeek and C. Schmid are with the LEAR team, Inria Grenoble RhôneAlpes, Laboratoire Jean Kuntzmann, CNRS, University Grenoble Alpes, France.E-mail: \{Jakob.Verbeek, cordelia.schmid\}@inria.fr.

Manuscript received 25 Dec. 2014; revised 8 Dec. 2015; accepted 15 Jan. 2016. Date of publication 25 Feb. 2016; date of current version 12 Dec. 2016.

Recommended for acceptance by G. Mori.

For information on obtaining reprints of this article, please send e-mail to: reprints@ieee.org, and reference the Digital Object Identifier below.

Digital Object Identifier no. 10.1109/TPAMI.2016.2535231 weakly supervised training, mixed fully and weakly supervised [9], active [52], and semi-supervised [40] learning and unsupervised object discovery [11] methods have also been explored to reduce the amount of labeled training data for object detector training. In active learning bounding box annotations are used, but requested only for images where the annotation is expected to be most effective. Semi-supervised learning, on the other hand, leverages unlabeled images by automatically detecting objects in them, and use those to better model the object appearance variations.

In this paper we consider WSL to learn object detectors from image-wide labels. We follow an MIL approach that interleaves training of the detector with re-localization of object instances on the positive training images. Following recent state-of-the-art work in fully supervised detection [13], [22], [50], we represent (tentative) detection windows using Fisher vectors (FVs) [39] and convolutional neural network (CNN) features [29]. As we explain in Section 3, when used in an MIL framework, the high-dimensionality of the window features makes MIL quickly convergence to poor local optima after initialization. Our main contribution is a multi-fold training procedure for MIL, which avoids this rapid convergence to poor local optima. A second novelty of our approach is the use of a "contrastive" background descriptor that is defined as the difference of a descriptor of the object window and a descriptor of the remaining image area. The score for this descriptor of a linear classifier can be interpreted as the difference of scores for the foreground and background. In this manner we direct the detector to learn the difference between foreground and background appearances. Finally, inspired from the objectness prior in [17], we propose a window refinement method that improves the weakly supervised localization accuracy by incorporating a category-independent objectness measure.

We present a detailed evaluation using the VOC 2007 dataset in Section 4. The experimental results show that our multi-fold MIL training improves performance for both FV 
and CNN features. We also show that WSL performance can be further improved by combining the two descriptor types and applying our window refinement method. The evaluation shows that our system obtains state-of-the-art results on VOC 2007. We also present results for VOC 2010 which was not yet used in previous work.

Part of the material presented here appeared in [14]. Besides a more detailed presentation and discussion of the most recent related work, the current paper extends it in several ways. We enhanced our WSL method by introducing a window refinement method. We also added additional experiments using CNN features, and their combination with FV features. Finally, we included experiments when training in a mixed supervision setting, where part of the images are weakly supervised and others are labeled with full bounding-box annotations.

\section{Related Work}

The majority of related work treats WSL for object detection as a multiple instance learning [18] problem. Each image is considered as a "bag" of examples given by tentative object windows. Positive images are assumed to contain at least one positive object instance window, while negative images only contain negative windows. The object detector is then obtained by alternating detector training, and using the detector to select the most likely object instances in positive images.

In many MIL problems, e.g., such as those for weakly supervised face recognition [6], [19], the number of examples per bag is limited to a few dozen at most. In contrast, there is a vast number of examples per bag in the case of object detector training since the number of possible object bounding boxes is quadratic in the number of image pixels. Candidate window generation methods, e.g., [1], [24], [49], [56], can be used to make MIL approaches to WSL for object localization manageable, and make it possible to use powerful and computationally expensive object models.

Although candidate window generation methods can significantly reduce the search space per image, the selection of windows across a large number of images is inherently a challenging problem, where an iterative WSL method can typically find only a local optimum depending on the initial windows. Therefore, in this section, we first overview the initialization methods proposed in the literature, and then summarize the iterative WSL approaches.

\subsection{Initialization Methods}

A number of different strategies to initialize the MIL detector training have been proposed in the literature. A simple strategy, e.g., taken in [28], [35], [38], is to initialize by taking large windows in positive images that (nearly) cover the entire image. This strategy exploits the inclusion structure of the MIL problem for object detection. That is: although large windows may contain a significant amount of background features, they are likely to include positive object instances.

Another strategy is to utilize a class-independent saliency measure that aims to predict whether a given image region belongs to an object or not. For example, Deselaers et al. [17] generate candidate windows using the objectness method [2] and assign per-window weights using a saliency model trained on a small set of non-target classes. Siva et al. [44] instead estimate an unsupervised patch-level saliency map for a given image by measuring the average similarity of each patch to the other patches in a retrieved set of similar images. In each image, an initial window is found by sampling from the corresponding saliency map.

Alternatively, a class-specific initialization method can be used. For example, Chum and Zisserman [12] select the visual words that predominantly appear in the positive training images and initialize WSL by finding the bounding box of these visual words in each image. Siva and Xiang [45] propose to initially select one of the candidate windows sampled using the objectness method at each image such that an objective function based on intra-class and interclass pairwise similarities is maximized. However, this formulation leads to a difficult combinatorial optimization problem. Siva et al. [43] propose a simplified approach where a candidate window is selected for a given image such that the distance from the selected window to its nearest neighbor among windows from negative images is maximal. Relying only on negative windows not only avoids the difficult combinatorial optimization problem, but also has the advantage that their labels are certain, and there is a larger number of negative windows available which makes the pairwise comparisons more robust.

Shi et al. [40] propose to estimate a per-patch class distribution by using an extended version of the Latent Dirichlet Allocation (LDA) [10] topic model. Their approach assigns object class labels across different object categories concurrently, which allows to benefit from explaining-away effects, i.e., an image region cannot be identified as an instance for multiple categories. The initial windows are then localized by sampling from the saliency maps.

Song et al. [46] propose a graph-based initialization method. The main idea is to select a subset of the candidate windows such that the nearest neighbors of the selected windows correspond to the candidate windows in the positive images, rather than the ones in the negative images. The approach is formulated as a discriminative submodular cover problem on the similarity graph of the windows. In a follow-up work, Song et al. [47] extend this approach to find multiple non-overlapping regions corresponding to object parts. The initial object windows are then generated by finding frequent part configurations and their bounding boxes.

\subsection{Iterative Learning Methods}

Once the initial windows are localized, typically an iterative learning approach is employed in order to improve the initial localizations in the training images.

One of the early examples of WSL for object detector training is proposed by Crandall and Huttenlocher [15]. In their work, object and part locations are treated as latent variables in a probabilistic model. These variables are automatically inferred and utilized during training using an Expectation Maximization (EM) algorithm. The main focus of their work, however, is on training a partbased object detector without using manual part annotations, rather than training in terms of image labels. Their approach is evaluated on datasets containing images with uncluttered backgrounds and little variance in terms of object locations, which is an unrealistic testbed for WSL of object detectors. 
Several WSL methods aim to localize objects via selecting a subset of candidate windows based on pairwise similarities. For example, Kim and Torralba [28] use a link analysis based clustering approach. Chum and Zisserman [12] iteratively select windows and update the similarity measure that is used to compare windows. The window selection is done by updating one image at a time such that the average pairwise similarity across the positive images is maximized. The similarity measure, which is defined in terms of bag-ofword (BoW) descriptors [16], is updated by selecting the visual words that predominantly appear in the selected windows rather than the negative images.

Deselaers et al. [17] propose a CRF-based model that jointly infers the object hypotheses across all positive training images, by exploiting a fully-connected graphical model that encourages visual similarity across all selected object hypotheses. Unlike the methods of [28] and [12], the CRFbased model additionally utilize a unary potential function that scores candidate windows individually based on their window descriptors and objectness scores. The parameters of the pairwise and unary potential functions are updated, and the positive windows are selected in an iterative fashion. Prest et al. [37] extend these ideas to weakly supervised detector training from videos by extracting candidate spatio-temporal tubes based on motion cues and by defining WSL potential functions over tubes instead of windows.

Our window refinement method is inspired from the use of an objectness model as a class-independent prior in [17]. While Deselaers et al. [17] use the objectness prior in all training iterations, we update the coordinates of the topscoring final localizations, using the local greedy search procedure from [56]. In addition, instead of using the objectness model in [2], we use the edge-driven objectness measure [56], which evalutes the alignment between each window and the edges around it.

Most recent work is predominantly based on iteratively selecting the highest scoring detections as the positive training examples and training the detection models. We refer to this approach as standard MIL. Using this approach, an offthe-shelf detector can be trained in a weakly supervised setting. For example, Nguyen et al. [34] and Blaschko et al. [9] train the branch-and-bound localization [31] based detectors over BoW descriptors in this manner. Blaschko et al. also investigate the use of object-center annotations as an alternative WSL setting.

The DPM model [21] has been utilized with standard MIL based training approaches by a number of other WSL approaches, see e.g., [35], [40], [43], [44], [45]. The majority of the works use the standard DPM training procedure and differ in terms of their initialization procedures. One exception is that Siva and Xiang [45] propose a method to detect when the iterative training procedure drifts to background regions. In addition, Pandey and Lazebnik [35] carefully study how to tune DPM training for WSL purposes. They propose to restrict each re-localization stage such that the bounding boxes between two iterations must meet a minimum overlap threshold, which avoids big fluctuations across the iterations. Moreover, they propose a heuristic to automatically crop windows with near-uniform backgrounds.

Russakovsky et al. [38] use a similar approach based on Locality-constrained Linear Coding descriptors [54] over the candidate windows generated using the Selective Search method [49]. They use a background descriptor computed over features outside the window, which helps to better localize the objects as compared to only modeling the windows themselves.

Song et al. [46] develop a smoothed version of the standard MIL approach using Nesterov's smoothing technique [33]. The main motivation is to increase robustness against incorrectly selected windows, particularly in early iterations, by training with multiple windows per positive image. The candidate windows are generated using selective search [49] and the window descriptors are extracted using the CNN model of [29].

Bilen et al. [8] propose an alternative smoothed version of standard MIL. Instead of selecting the top scoring window in a positive image, they propose to train over all windows that are weighted by a soft-max function over the classification scores. In addition, they utilize additional regularization terms that aim to (i) enforce that positive training windows and their horizontal mirrors score similarly and, (ii) avoid obtaining high classification scores for multiple classes for a single window. They also utilize selective search candidate windows [49] and CNN features [29].

Recently, Wang et al. [53] propose a two-step method, which first groups selective search candidate windows [49] from the positive images of a class into visual clusters and then chooses the most discriminative cluster of windows. In the first step, the CNN features [29] are clustered using probabilistic latent semantic analysis (PLSA) [25]. In the second step, for each visual cluster, image descriptors are extracted from the CNN-based window descriptors of the windows associated with the cluster. Finally, one visual cluster for each class is selected based on the image classification performance of the corresponding image descriptors.

Our approach is most related to that of Russakovsky et al. [38]. We also rely on the selective search windows [49], and use a similar initialization strategy. A critical difference from [38] and other WSL approaches based on iterative detector training, however, is our multi-fold MIL training procedure which we describe in the next section. Our multifold MIL approach is also related to the work of Singh et al. [42] on unsupervised vocabulary learning for image classification. Starting from an unsupervised clustering of local patches, they iteratively train SVM classifiers on a subset of the data, and evaluate it on another set to update the training data from the second set.

We note that avoiding poor local optima in training of models with non-convex objectives is a fundamental problem in machine learning, and there are many aspects of it. For example, curriculum learning (CL) [5], which is a conceptual framework, suggests that training can be improved by initializing a model with easy examples, and then, gradually utilizing more complex ones. Kumar et al. [30] propose a CL formulation for latent variable models by considering the loss function as a measure of example difficulty, which excludes low-scoring examples in early training iterations. Progressively increasing the latent search space can also be interpreted as a CL approach to avoid making unstable inferences in early iterations, see e.g., [7], [38]. Although our work is related, our focus is different in the sense that we target the problem of 
degenerate latent variable inference due to use of highdimensional descriptors.

\section{Weakly Supervised ObJect Localization}

Below, we present our multi-fold MIL approach in Section 3.2 and window refinement method in Section 3.3, but first briefly describe our FV and CNN based object appearance descriptors.

\subsection{Features and Detection Window Representation}

In our experiments we rely on FV and $\mathrm{CNN}$ based representations. In either case, we use the selective search method of Uijlings et al. [49]. It generates a limited set of around 1,500 candidate windows per image. This speeds-up detector training and evaluation, while filtering out the most implausible object locations.

The FV-based representation is based on our previous work [13] for fully supervised detection. In particular, we aggregate local SIFT descriptors into an FV representation to which we apply $\ell_{2}$ and power normalization [39]. We concatenate the FV computed over the full detection window, and $16 \mathrm{FVs}$ computed over the cells in a $4 \times 4$ grid over the window, inspired by the spatial pyramid representation of Lazebnik et al. [32]. Using PCA to project the SIFTs to 64 dimensions, and a mixture of Gaussians (MoG) of 64 components, this yields a descriptor of 140,352 dimensions. We reduce the memory footprint, and speed up our iterative training procedure, by using the $\mathrm{PQ}$ and Blosc feature compression [3], [26].

Similar to Russakovsky et al. [38], we add contextual information from the part of the image not covered by the window. Full-image descriptors, or image classification scores, are commonly used for fully supervised object detection, see e.g., [13], [48]. For WSL, however, it is important to use the complement of the object window rather than the full image, to ensure that the context descriptor also depends on the window location. This prevents learning degenerate detection models, since otherwise the context descriptor can be used to perfectly separate the training images regardless of the object localization.

To enhance the effectiveness of the context descriptor we propose a "contrastive" version, defined as the difference between the background FV $x_{b}$ and the $1 \times 1$ foreground FV $x_{f}$. Since we use linear classifiers, the contribution to the window score of this descriptor, given by $w^{\top}\left(x_{b}-x_{f}\right)$, can be decomposed as a sum of a foreground and a background score: $\boldsymbol{w}^{\top} x_{b}$ and $-\boldsymbol{w}^{\top} x_{f}$ respectively. Because the foreground and background descriptor have the same weight vector, up to a sign flip, we effectively force features to either score positively on the foreground and negatively on the background, or vice-versa within the contrastive descriptor. This prevents the detector to score the same features positively on both the foreground and the background.

To ensure that we have enough SIFT descriptors for the background $\mathrm{FV}$, we filter the detection windows to respect a margin of at least 4 percent from the image border, i.e. for a $100 \times 100$ pixel image, windows closer than four pixels to the image border are suppressed. This filtering step removes about half of the windows. We initialize the MIL training with the window that covers the image, up to a 4

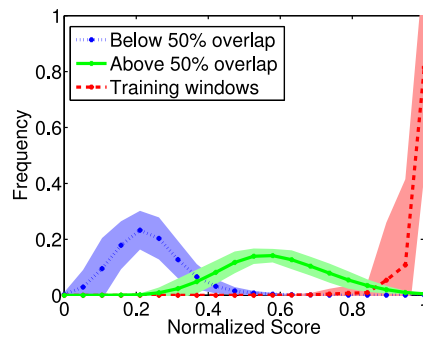

(a) Fisher vectors

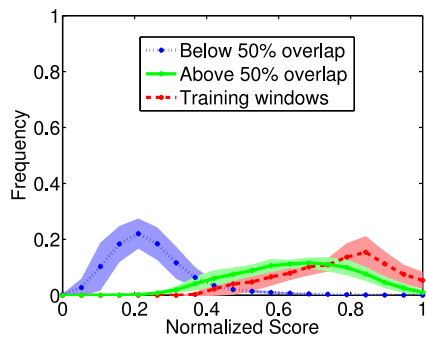

(b) CNN features
Fig. 1. Distribution of the window scores in the positive training images after the fifth iteration of standard MIL training on VOC 2007 for FVs (left) and CNNs (right). For each figure, the right-most curve corresponds to the windows chosen in the most recent re-localization step and used for training the detector. The curve in the middle corresponds to the other windows that overlap more than 50 percent with the training windows. Similarly, the left-most curve corresponds to the windows that overlap less than 50 percent. Each curve is obtained by averaging all per-class score distributions. The surrounding regions show the standard deviation.

percent margin, so that all instances are captured by the initial windows.

We extract the $\mathrm{CNN}$ features using the $\mathrm{CNN}$ architecture of Krizhevsky et al. [29]. We utilize the first seven layers of the CNN model, which consists of five convolutional and two fully-connected layers. The CNN model is pre-trained on the ImageNet ILSVRC 2012 dataset using the Caffe framework [27]. Following Girshick et al. [22], we crop and resize the mean-subtracted regions corresponding to the candidate windows to images of size $224 \times 224$, as required by the CNN model. Finally, we apply $\ell_{2}$ normalization to the resulting 4,096 dimensional descriptors.

An important advantage of the CNN features is that some of the feature dimensions correspond to higher level image structures, such as certain animal faces and bodies [22], which can simplify the WSL problem. Our experimental results show that the $\mathrm{CNN}$ features perform better than the FV features, but that they are complementary since best performance is obtained when combining both features.

\subsection{Weakly Supervised Object Detector Training}

The dominant method for weakly supervised training of object detectors is the standard MIL approach, which is based on iterating between the training and the relocalization stages, as described in Section 2.2. Note that in this approach, the detector used for re-localization in positive images is trained using positive samples that are extracted from the very same images. Therefore, there is a bias towards re-localizing on the same windows; in particular when high capacity classifiers are used which are likely to separate the detector's training data. For example, when a nearest neighbor classifier is used the relocalization will be degenerate and not move away from its initialization, since the same window will be found as its nearest neighbor.

The same phenomenon occurs when using powerful and high-dimensional image representations to train linear classifiers. We illustrate this in Fig. 1, which shows the distribution of the window scores in a typical standard MIL iteration. We observe that the windows used in SVM training score significantly higher than the other ones, including those with a significant spatial overlap with the most recent 

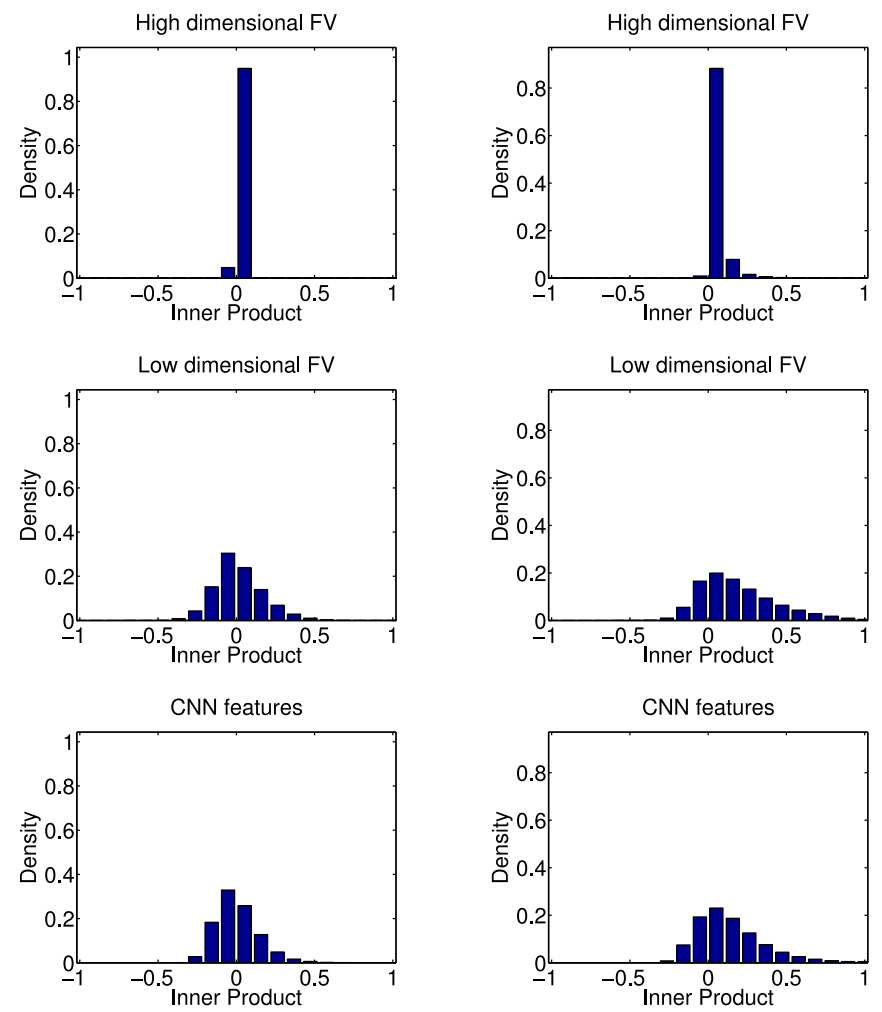

(a) All pairs

(b) Within-image pairs.

Fig. 2. Distribution of inner products, scaled to the interval $[-1+1]$, of pairs of 25,000 windows sampled from 250 images using our highdimensional FV (top), a low-dimensional FV (middle), and CNN features (bottom). (a) uses all window pairs and (b) uses only within-image pairs, which are more likely to be similar.

training windows, especially when the high-dimensional FV descriptors are used.

As a result, standard MIL typically results in degenerate relocalization. This problem is related to the dimensionality of the window descriptors. We illustrate this in Fig. 2, where we show the distribution of inner products between the descriptors of different windows. In Fig. 2a, we use random window pairs within and across images. In Fig. 2b, we use only withinimage pairs, which are more likely to be similar, and therefore the histograms models are shifted slightly to larger values. We show the distributions using both our 140,352 dimensional FVs, 516 dimensional FVs obtained using four Gaussians without spatial grid, and 4,096 dimensional CNN-based descriptors. ${ }^{1}$ Unlike in the case of low-dimensional FVs or CNN-based descriptors, almost all window descriptors are near orthogonal in the high-dimensional FV case even when we use within-image pairs only. Also, recall that the weight vector of a standard linear SVM classifier can be written as a linear combination of training samples, $w=\sum_{i} \alpha_{i} x_{i}$. Therefore, the training windows are likely to score significantly higher than the other windows in positive images in the highdimensional case, resulting in degenerate re-localization behavior. In Section 4, we verify this hypothesis experimentally by comparing the localization behavior using the lowdimensional versus the high-dimensional descriptors.

1. To make the histograms comparable, we make all descriptors zero mean, before $\ell_{2}$ normalization, and computing the inner products.
Note that increasing regularization weight in SVM training does not remedy this problem. The $\ell_{2}$ regularization term with weight $\lambda$ restricts the linear combination weights such that $\left|\alpha_{i}\right| \leq 1 / \lambda$. Therefore, although we can reduce the influence of individual training samples via regularization, the resulting classifier remains biased towards the training windows since the classifier is a linear combination of the window descriptors. In Section 4, we verify this hypothesis by evaluating the regularization weight's effect on the localization performance.

To address this issue-without sacrificing the descriptor dimensionality, which would limit its descriptive powerwe propose to train the detector using a multi-fold procedure, reminiscent of cross-validation, within the MIL iterations. We divide the positive training images into $K$ disjoint folds, and re-localize the images in each fold using a detector trained using windows from positive images in the other folds. In this manner the re-localization detectors never use training windows from the images to which they are applied. Once re-localization is performed in all positive training images, we train another detector using all selected windows. This detector is used for hard-negative mining on negative training images, and returned as the final detector.

We summarize our multi-fold MIL training procedure in Algorithm 1. The standard MIL algorithm that does not use multi-fold training does not execute steps 2(a) and 2(b), and re-localizes based on the detector learned in step 2(c).

\section{Algorithm 1. Multi-fold Weakly Supervised Training}

1) Initialization: positive and negative examples are set to entire images up to a $4 \%$ border

2) For iteration $t=1$ to $T$

a) Divide positive images randomly into $K$ folds

b) For $k=1$ to $K$

i) Train using positive examples in all folds but $k$, and all negative examples

ii) Re-localize positives by selecting the top scoring window in each image of fold $k$ using this detector

c) Train detector using re-localized positives and all negatives

d) Add new negative windows by hard-negative mining 3) Return final detector and object windows in train data

The number of folds used in our multi-fold MIL training procedure should be set to strike a good trade-off between two competing factors. On the one hand, using more folds increases the number of training samples per fold, and is therefore likely to improve re-localization performance. On the other hand, using more folds increases the computational cost. We experimentally analyze this trade-off in Section 4.

\subsection{Window Refinement}

We now explain our window refinement method. It updates the localizations obtained by the last multi-fold MIL iteration. The final detector is, then, re-trained based on these refinements.

An inherent difficulty for weakly supervised object localization is that WSL labels only permit to determine the most repeatable and discriminative patterns for each class. Therefore, even though the windows found by WSL are likely to 
overlap with target object instances, it can not be ensured that they will delineate object boundaries.

To better take into account object boundaries, we use the edge-driven objectness measure of Zitnick and Dollar [56]. The main idea in [56] is to score a given window based on the number of contours that are fully contained inside the window, with an increased weight on near-boundary edge pixels. Thus, windows that tightly enclose long contours are scored highly, whereas those with predominantly straddling contours are penalized. Additionally, in order to reduce the effect of marginal misalignments, the coordinates of a given window are updated using a greedy local search procedure that aims to increase the objectness score. In [56], the objectness measure is used for generating object proposals. For this purpose, a set of initial windows are first generated using a sliding window mechanism, and then, updated and scored using the local search procedure. The final windows are obtained by applying a non-maxima suppression procedure.

We instead use the edge-driven objectness measure to improve our WSL outputs. For this purpose, we combine the objectness measure with the classification scores given by multi-fold MIL. More specifically, we first utilize the local search procedure in order to update and score the candidate detection windows based on the objectness measure, without updating the classification scores. To make the classification and objectness scores comparable, we scale each score channel to the range $[0,1]$ for all windows in the positive training images. We, then, combine linearly the classification and objectness scores with equal weights, and select the top detection in each image with respect to this combined score. In order to avoid selecting the windows irrelevant for the target class, but with a high objectness score, we restrict the search space to the top- $N$ windows per image in terms of the classification score. While we use $N=10$ in all our experiments, we have empirically observed that the refinement method significantly improves the localization results for $N$ ranging from 1 to 50. The improvement is comparable for $N \geq 5$.

In Fig. 3, we show two example images for the classes horse and $\operatorname{dog}$ in the left column, together with the corresponding edge maps in the right column. In these images, the dashed (pink) boxes show the output of multi-fold MIL training and the solid (yellow) boxes show the outputs of the window refinement procedure. Even though the initial windows are located on the object instances, they are evaluated as incorrect due to the low overlap ratios with the ground-truth ones. The edge maps show that many contours, i.e., most object contours, straddle the initial window boundaries. In contrast, the corrected windows have higher percentages of fully contained contours, i.e., the contours relevant for the objects.

The refined windows are likely to be better aligned with object instances. Thus, their horizontal mirrors are more reliable and can be used as additional training examples. We evaluate the impact of window refinement and flipped examples in the next section.

\section{EXPERIMENTAL EVALUATION}

In this section we present a detailed analysis and evaluation of our weakly supervised localization approach.
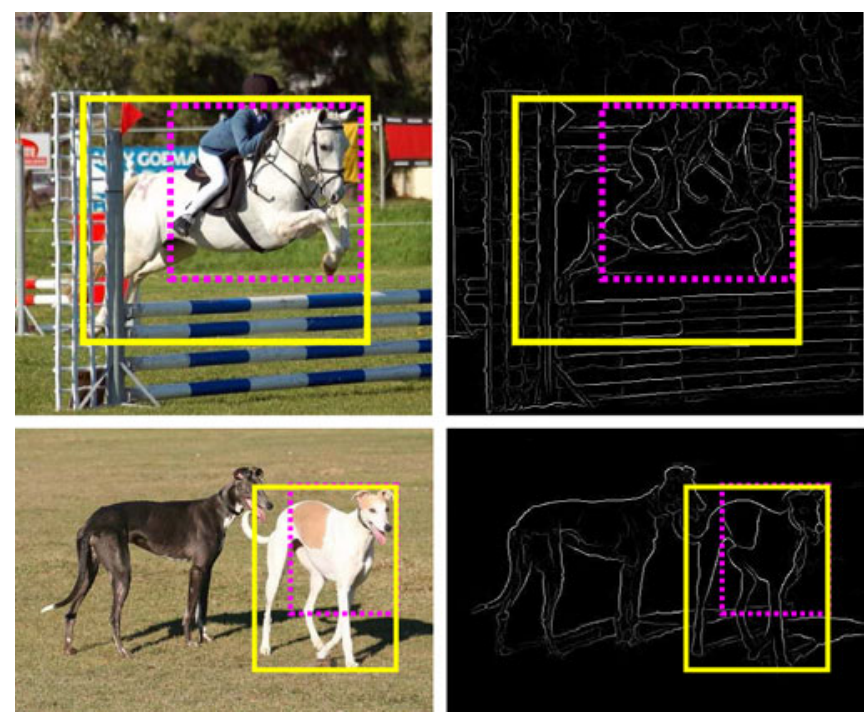

Fig. 3. Illustration of our window refinement. Dashed boxes (pink) show the localization before refinement, and the solid boxes (yellow) show the result of the window refinement method. The images on the right show the edge maps that are used to compute the objectness measure.

\subsection{Dataset and Evaluation Criteria}

We use the PASCAL VOC 2007 and 2010 datasets [20] in our experiments. Most of our experiments use the 2007 dataset, which allows us to compare to previous work. To the best of our knowledge, we are the first to report WSL performance on the VOC 2010 dataset. Following [17], [35], [40], during training we discard any images that only contain object instances marked as "difficult" or "truncated". During testing all images are included. We use linear SVM classifiers, and set the weight of the regularization term and the class weighting to fixed values based on preliminary experiments. We perform two hard-negative mining steps [21] after each re-localization phase. Finally, while we run all experiments using the same random seed, we have empirically verified that changing the seed does not affect the final detection performance significantly.

Following [17], we assess performance using two measures. First, we evaluate the fraction of positive training images in which we obtain correct localization (CorLoc). Second, we measure the final object detection performance on the test images using the standard protocol [20], average precision (AP) per class and mean AP (mAP) across all classes. For both measures, we consider that a window is correct if it has an intersection-over-union ratio of at least 50 percent with a ground-truth object. Since CorLoc is not consistently measured across studies due to changes in training sets, we use CorLoc mainly as a diagnostic measure, and use AP to compare to the state-of-the-art.

\subsection{Multi-Fold MIL Training and Features}

In our first experiment, we compare (a) standard MIL training, and (b) our multi-fold MIL algorithm with $K=10$ folds. Both are initialized from the full image up to the 4 percent boundary. We also consider the effectiveness of background features for the FV representation. We test three variants: (F) foreground only descriptor, (B) an FV computed over the window background, and (C) our contrastive background descriptor. Finally, we compare the FV 
TABLE 1

Weakly Supervised Learning Using FV and CNN Features, Measured in Terms of Correct Localization (CorLoc) Measure on VOC 2007 Training Set

\begin{tabular}{|c|c|c|c|c|c|c|c|c|c|c|c|c|c|c|c|c|c|c|c|c|c|}
\hline \multicolumn{22}{|c|}{ standard MIL } \\
\hline $\mathrm{V}: \mathrm{F}$ & 46.2 & 32.2 & 32.0 & 24.1 & 4.0 & 45.1 & 51.5 & 37.6 & 6.8 & 24.3 & 14.3 & 43.0 & 36.2 & 52.7 & 19.3 & 9.3 & 20.3 & 24.5 & 45.1 & 14.2 & 29.1 \\
\hline $\mathrm{F}: \mathrm{F}+\mathrm{B}$ & 50.3 & 32.2 & 32.4 & 24.8 & 4.0 & 45.1 & 2.2 & 41.1 & 6.8 & 25.2 & 14.3 & 44.1 & 38.2 & 53.7 & 20.5 & 9.3 & 20.3 & 24.5 & 43.4 & 14.2 & 29.8 \\
\hline $\mathrm{V}: \mathrm{F}+\mathrm{C}$ & 48.6 & 32.8 & 30.9 & 25.5 & 4.0 & 43.4 & 52.2 & 40.6 & 6.8 & 27.2 & 14.3 & 43.7 & 38.6 & 52.7 & 20.0 & 8.8 & 20.3 & 24.5 & 45.1 & 14.7 & 29.7 \\
\hline $\mathrm{NN}$ & 54.3 & 55.6 & 49.5 & 31.7 & 15.9 & 61.5 & 72.2 & 33.2 & 16.5 & 43.7 & 22.4 & 34.8 & 58.5 & 64.4 & 25.1 & 31.9 & 36.2 & 34.0 & 52.2 & 31.5 & 41.2 \\
\hline $\mathrm{FV}+\mathrm{CNN}$ & 49.1 & 36.1 & 38.9 & 30.3 & 5.1 & 49.2 & 62.4 & 47.5 & 6.8 & 35.0 & 18.4 & 44.8 & 45.4 & 54.3 & 29.3 & 13.2 & 26.1 & 29.2 & 48.7 & 18.8 & 34.4 \\
\hline \multicolumn{22}{|c|}{ multi-fold MIL } \\
\hline $\mathrm{V}: \mathrm{F}$ & 48.0 & 55.6 & 25.8 & 4.1 & 6.3 & 53.3 & & 23.3 & 8.8 & 57.3 & 4.1 & 27.6 & 52.7 & 66.0 & 33.2 & 15.4 & 55.1 & 14.2 & 49.6 & 62.4 & 36. \\
\hline$V: F+B$ & 55.5 & 56.1 & 21.8 & 27.6 & 4.5 & 51.6 & 66.5 & 19.3 & 8.4 & 59.2 & 2.0 & 26.2 & 56.0 & 64.9 & 35.5 & 20.9 & 58.0 & 10.4 & 56.6 & 59.4 & 38. \\
\hline $\mathrm{V}: \mathrm{F}+\mathrm{C}$ & 56.6 & 58.3 & 28.4 & 20.7 & 6.8 & 54.9 & 69.1 & 20.8 & 9.2 & 50.5 & 10.2 & 29.0 & 58.0 & 64.9 & 36.7 & 18.7 & 56.5 & 13.2 & 54.9 & 59.4 & 38.8 \\
\hline CNN & 53.2 & 66.7 & 51.3 & 31.7 & 19.3 & 70.5 & 72.0 & 23.3 & 24.9 & 62.1 & 32.7 & 28.0 & 54.6 & 64.9 & 22.1 & 39.0 & 55.1 & 33.0 & 54.9 & 40.1 & 45.0 \\
\hline $\mathrm{FV}+\mathrm{CNN}$ & 57.2 & 62.2 & 50.9 & 37.9 & 23.9 & 64.8 & 74.4 & 24.8 & 29.7 & 64.1 & 40.8 & 37.3 & 55.6 & 68.1 & 25.5 & 38.5 & 65.2 & 35.8 & 56.6 & 33.5 & 47.3 \\
\hline
\end{tabular}

We compare foreground $(F)$, background $(B)$ and contrastive background $(C) F V$ s. Contrastive background is used in the FV $+C N N$ combination.

representation to the $\mathrm{CNN}$ representation and the $\mathrm{FV}+\mathrm{CNN}$ combination (by means of concatenating the descriptors). Together, this yields ten combinations of features and training algorithms. Table 1 presents results in terms of CorLoc on the training set, and Table 2 presents results in terms of AP on the test set.

From the results we see that, averaged over the classes, multi-fold MIL outperforms standard MIL for all five tested representations, and for both CorLoc and AP. Furthermore, we see that the CorLoc differences across different FV descriptors are rather small when using standard MIL training. This is due to the degenerate re-localization performance with high-dimensional descriptors for standard MIL training as discussed in Section 3.2; we will come back to this point below. For multi-fold training, the CNN features give better results than FV for 12 and 13 classes in terms of CorLoc and AP, respectively. They also benefit significantly from our multi-fold training procedure, although to a lesser extent than the FV. This is due to the lower dimensionality of the CNN features compared to the FV features.

While the CNN features give better performance overall than $\mathrm{FV}$, we observe that the $\mathrm{FV}+\mathrm{CNN}$ feature combination improves over the individual features in 13 of 20 classes in terms of both CorLoc and AP scores using multi-fold MIL. Importantly, we note that standard MIL over the combined feature space performs poorly at 34.4 percent CorLoc and 22.0 percent mAP compared to 47.3 percent CorLoc and 27.4 percent $\mathrm{mAP}$ for multi-fold MIL.

Fig. 4 presents examples of re-localization using standard and multi-fold MIL training. In all three cases, we observe that standard MIL gets stuck with the windows found by the first re-localization step. In contrast, multi-fold MIL is able to progressively localize down to smaller image regions. In the bicycle and motorbike examples, multi-fold MIL successfully localizes the object instances. In the cat example, on the other hand, while the window localized by standard MIL is correct, multi-fold MIL localizes the cat face, which has below 50 percent overlap with the object bounding box. The failure example in Fig. 4 affirms the difficulty for weakly supervised localization that we have pointed out in Section 3.3: the WSL labels only indicate to learn a model for the most repeatable structure in the positive training images. For the cat class, due to the highly deformable body, the head can be argued to be the most distinctive and reliably detectable structure.

TABLE 2

Weakly Supervised Learning Using FV and CNN Features, Measured in Terms of Average Precision (AP) Measure on VOC 2007 Test Set

\begin{tabular}{|c|c|c|c|c|c|c|c|c|c|c|c|c|c|c|c|c|c|c|c|c|c|}
\hline \multicolumn{22}{|c|}{ standard MIL } \\
\hline $\mathrm{FV}: \mathrm{F}$ & 5.4 & 31.9 & 5.6 & 2.3 & 0.2 & 27.9 & 35.4 & 20.6 & 0.5 & 6.8 & 4.9 & 14.0 & 17.0 & 35.2 & 7.1 & 6.2 & 5.8 & 5.1 & 20.7 & 8.1 & 14.0 \\
\hline $\mathrm{FV}: \mathrm{F}+\mathrm{B}$ & 28.8 & 30.7 & 10.5 & 6.6 & 0.3 & 30.1 & 36.2 & 22.7 & 0.9 & 7.2 & 3.4 & 16.3 & 22.3 & 35.5 & 7.7 & 9.2 & 7.5 & 3.9 & 26.2 & 6.5 & 15.6 \\
\hline $\mathrm{FV}: \mathrm{F}+\mathrm{C}$ & 26.1 & 31.6 & 8.3 & 5.3 & 1.3 & 31.1 & 36.9 & 22.7 & 0.7 & 7.7 & 2.1 & 16.6 & 24.5 & 36.7 & 7.7 & 4.7 & 4.2 & 4.5 & 30.0 & 7.5 & 15.5 \\
\hline CNN & 34.2 & 39.9 & 26.5 & 11.7 & 7.0 & 38.0 & 45.6 & 19.6 & 6.2 & 25.5 & 5.3 & 18.8 & 34.2 & 42.3 & 15.6 & 20.0 & 18.6 & 23.5 & 37.0 & 15.8 & 24.3 \\
\hline $\mathrm{FV}+\mathrm{CNN}$ & 36.4 & 31.7 & 23.9 & 11.7 & 1.5 & 37.8 & 40.4 & 29.4 & 1.1 & 17.1 & 5.1 & 29.0 & 32.3 & 40.9 & 15.2 & 8.2 & 14.3 & 19.7 & 36.9 & 8.2 & 22.0 \\
\hline \multicolumn{22}{|c|}{ multi-fold MIL } \\
\hline$V: F$ & 29.4 & 37.8 & 7.3 & 0.5 & 1.1 & 33.2 & & 14.3 & 1.0 & 21. & 9.2 & 9.4 & 29.1 & 37.3 & 15.5 & 9.8 & 27.9 & 4.7 & 29.4 & & 20.0 \\
\hline $\mathrm{FV}: \mathrm{F}+\mathrm{B}$ & 36.7 & 39.2 & 8.2 & 10.4 & 1.9 & 31.4 & 40.4 & 15.7 & 1.6 & 22.6 & 5.8 & 7.4 & 29.1 & 40.9 & 18.9 & 10.4 & 27.3 & 2.9 & 30.1 & 38.2 & 21.0 \\
\hline $\mathrm{FV}: \mathrm{F}+\mathrm{C}$ & 35.8 & 40.6 & 8.1 & 7.6 & 3.1 & 35.9 & 41.8 & 16.8 & 1.4 & 23.0 & 4.9 & 14.1 & 31.9 & 41.9 & 19.3 & 11.1 & 27.6 & 12.1 & 31.0 & 40.6 & 22.4 \\
\hline CNN & 32.1 & 46.9 & 28.4 & 12.0 & 9.6 & 39.4 & 45.5 & 16.2 & 14.8 & 33.1 & 11.6 & 14.0 & 31.2 & 39.3 & 13.1 & 19.7 & 30.5 & 23.4 & 37.0 & 19.6 & 25.9 \\
\hline $\mathrm{FV}+\mathrm{CNN}$ & 38.1 & 47.6 & 28.2 & 13.9 & 13.2 & 45.2 & 48.0 & 19.3 & 17.1 & 27.7 & 17.3 & 19.0 & 30.1 & 45.4 & 13.5 & 17.0 & 28.8 & 24.8 & 38.2 & 15.0 & 27.4 \\
\hline
\end{tabular}

We compare foreground (F), background (B) and contrastive background (C) FVs. Contrastive background is used in the FV+CNN combination. 


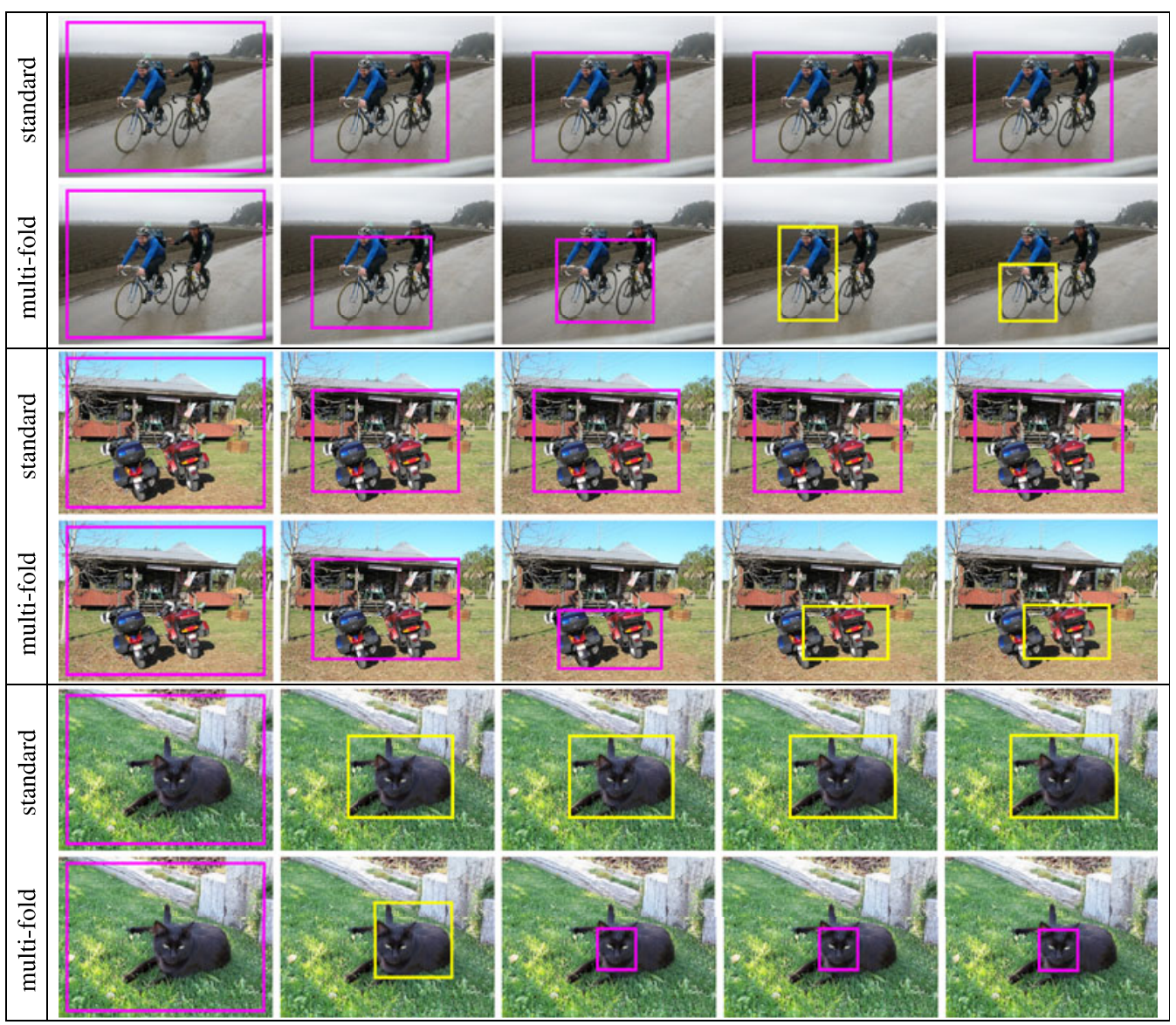

Fig. 4. Re-localization using standard and multi-fold MIL for images of the classes bicycle, motorbike, and cat from initialization (left) to the final localization (right) and three intermediate iterations based on $\mathrm{FV}(\mathrm{F}+\mathrm{C})$ descriptors. Correct localizations are shown in yellow, incorrect ones in pink.

This is what multi-fold MIL learns, but it degrades its CorLoc and AP scores. Parkhi et al. [36] also observed this, and proposed to localize cats and dogs based on a head detector in a fully supervised detector setting. Our window refinement method, which we evaluate below, resolves this issue to some extent.

In our next experiment, we further investigate the localization performances of the algorithms in terms of CorLoc across the training iterations. In the left panel of Fig. 5 we show the results for standard MIL, and our multi-fold MIL algorithm using 2, 10, and 20 folds. The results clearly show the degenerate re-localization performance obtained with standard MIL training, of which CorLoc stays (almost) constant in the iterations following the first re-localization stage. Our multi-fold MIL approach leads to substantially better performance, and ten MIL iterations suffice for the performance to stabilize. Results increase significantly by using 2 -fold and 10 -fold training respectively. The gain by using 20 folds is limited, however, and therefore we use 10 folds in the remaining experiments. We also include experiments with the 516 dimensional FV obtained using a 4-component MoG model, to verify the hypothesis of Section 3.2. The latter conjectured that the degenerate re-localization observed for standard MIL training is due to the trivial separability obtained for high-dimensional descriptors. Indeed, the lowest two curves in the left panel of Fig. 5 show that for this descriptor we obtain non-degenerate re-localization using standard MIL similar to multi-fold MIL. The performance is poor, however, due to limited representative power of the low-dimensional FVs.

In the middle panel of Fig. 5, we compare standard MIL and multi-fold MIL using the $\mathrm{CNN}$ features. We observe
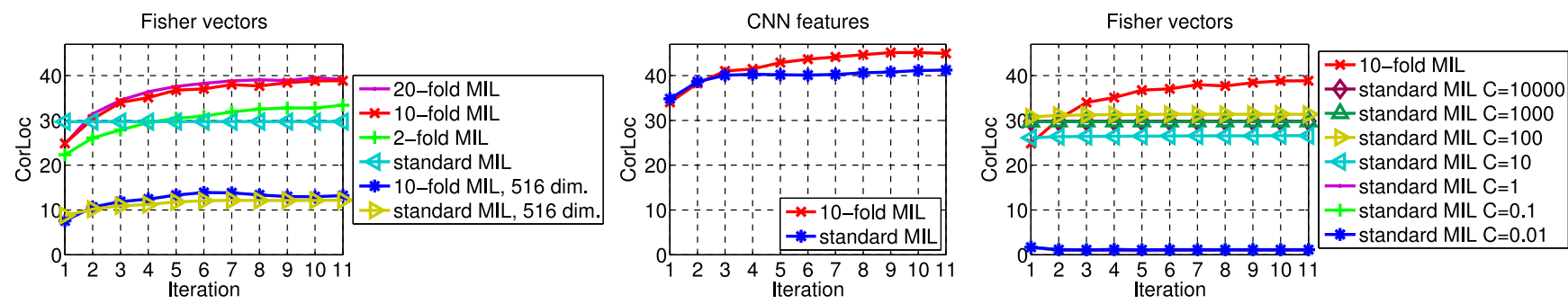

Fig. 5. Correct localization (CorLoc) performance (in the training set) over the MIL iterations, averaged across VOC 2007 classes. We show results for the high and low dimensional FVs (left panel), and the CNN features (middle panel). In the right panel, we compare 10-fold training with standard MIL training using different values of the SVM cost parameter $(C)$ for the high-dimensional FVs. 


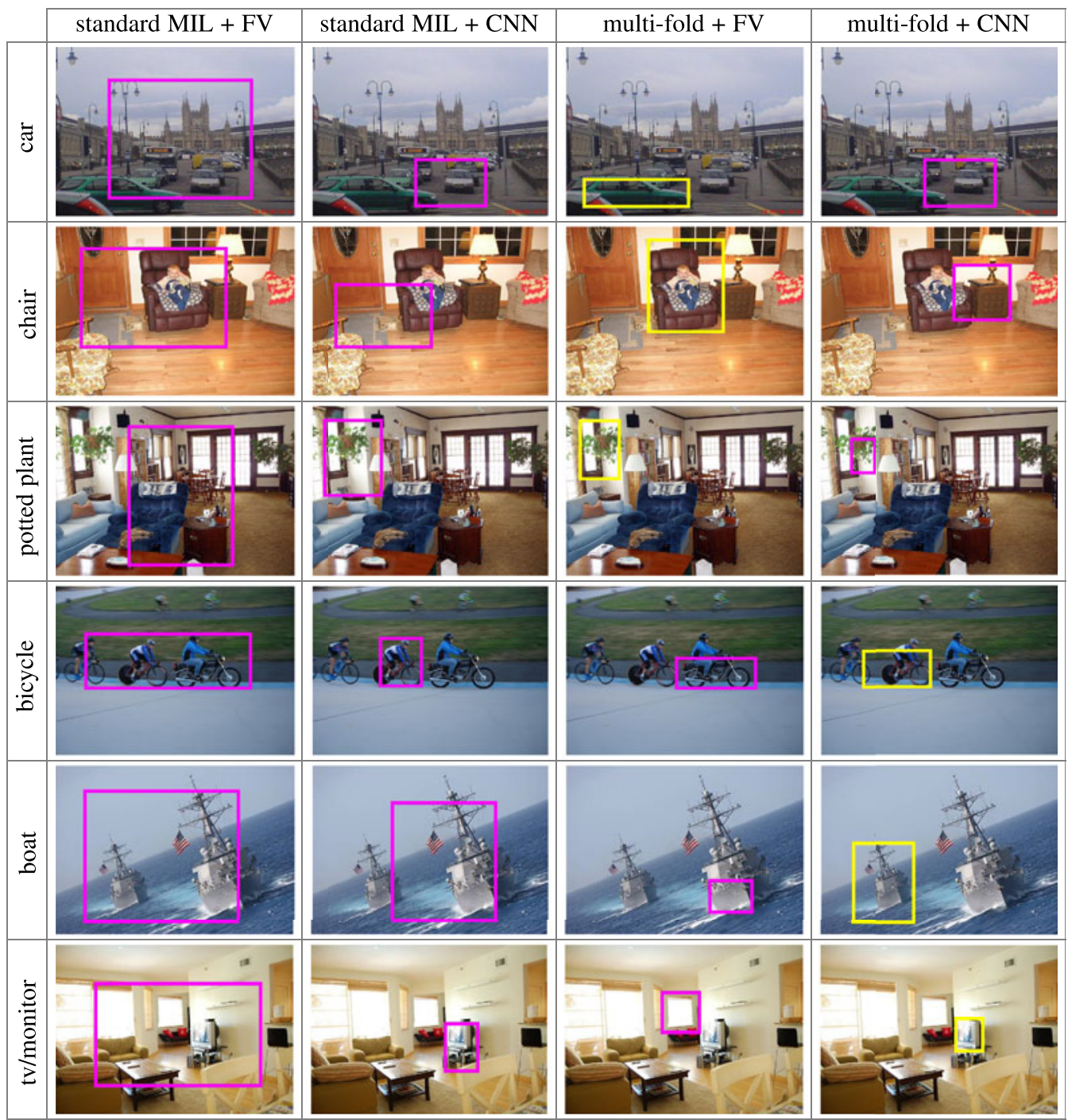

Fig. 6. Example localization results on the training images for standard MIL and multi-fold MIL algorithms with high-dimensional FV and CNN features. Correct localizations are shown in yellow, incorrect ones in pink.

that standard MIL is less affected by degenerate re-localization problem, compared to the case for high-dimensional FVs. This is in accordance with our observations for lowdimensional FVs, as the $\mathrm{CNN}$ features have an intermediate dimensionality of 4,096. Nevertheless, multi-fold MIL leads to significant improvements over the iterations, which results in 43.8 percent CorLoc, compared to 40.3 percent CorLoc for standard MIL.

The degenerate re-localization of standard MIL using highdimensional descriptors can be interpreted as over-fitting to the training data at an early stage. Therefore, the question is whether we can improve standard MIL by carefully tuning the trade-off between the regularization terms and the loss functions for SVM training. In the right panel of Fig. 5, we investigate this question by evaluating the standard MIL approach for different values of the cost parameter $(C)$ using high-dimensional FVs. The results show that, although choosing a proper $C$ value is important, it is not possible to solve the degenerate re-localization problem of standard MIL in this manner. Whereas using a too low $C$ value $(C \leq 1)$ causes standard MIL to drift off to a poor solution, larger $C$ values $(C \geq 10)$ result in degenerate re-localization.

\subsection{Evaluation of Window Refinement}

In Fig. 6 we provide examples of the localization results on the training images using standard and multi-fold MIL for FV and CNN features. The first three examples (car, chair, and potted plant) are only correctly localized using multifold MIL with FVs. These examples demonstrate the ability of our multi-fold training procedure to handle cases with multiple instances that appear in near proximity and with considerable background clutter. The last three examples (bicycle, boat, and to/monitor) are only correctly localized using multi-fold MIL with CNNs. In the bicycle example, we observe that multi-fold MIL with FVs mistakes a visually similar motorbike for a bicycle. Likewise, in the tv/monitor example, multi-fold MIL over FVs localizes a window that looks similar to a bright monitor. These examples suggest that (i) FV and CNN features can be complimentary to each other, which gives an insight for the success of the FV $+\mathrm{CNN}$ representation, and (ii) that some near-miss localizations might be corrected by a window refinement method.

We present the CorLoc and AP results for the window refinement method in Tables 3 and 4, respectively. All reported results are based on multi-fold training. The upper 
TABLE 3

Evaluation of Window Refinement on the VOC 2007 Dataset, in Terms of Training Set Localization Accuracy (CorLoc)

\begin{tabular}{|c|c|c|c|c|c|c|c|c|c|c|c|c|c|c|c|c|c|c|c|c|c|}
\hline & aero & bicy & bird & boa & bot & bus & car & cat & cha & cow & dtab & $\operatorname{dog}$ & hors & mbik & pers & plnt & she & sofa & trai & tv & Av. \\
\hline FV & 56.6 & 58.3 & 28.4 & 20.7 & 6.8 & 54.9 & 69.1 & 20.8 & 9.2 & 50.5 & 10.2 & 29.0 & 58.0 & 64.9 & 36.7 & 18.7 & 56.5 & 13.2 & 54.9 & 59.4 & 38.8 \\
\hline $\mathrm{CNN}$ & 53.2 & 66.7 & 51.3 & 31.7 & 19.3 & 70.5 & 72.0 & 23.3 & 24.9 & 62.1 & 32.7 & 28.0 & 54.6 & 64.9 & 22.1 & 39.0 & 55.1 & 33.0 & 54.9 & 40.1 & 45.0 \\
\hline $\mathrm{FV}+\mathrm{CNN}$ & 57.2 & 62.2 & 50.9 & 37.9 & 23.9 & 64.8 & 74.4 & 24.8 & 29.7 & 64.1 & 40.8 & 37.3 & 55.6 & 68.1 & 25.5 & 38.5 & 65.2 & 35.8 & 56.6 & 33.5 & 47.3 \\
\hline \multicolumn{22}{|c|}{ after window refinement } \\
\hline FV & 62.4 & 62.2 & 40.7 & 35.2 & 5.1 & 67.2 & 76.9 & 33.2 & 12.9 & 63.1 & 16.3 & 39.4 & 62.8 & 67.6 & 37.2 & 22.5 & 63.8 & 22.6 & 65.5 & 65.5 & 46.1 \\
\hline CNN & 67.1 & 66.1 & 49.8 & 34.5 & 23.3 & 68.9 & 83.5 & 44.1 & 27.7 & 71.8 & 49.0 & 48.0 & 65.2 & 79.3 & 37.4 & 42.9 & 65.2 & 51.9 & 62.8 & 46.2 & 54.2 \\
\hline $\mathrm{FV}+\mathrm{CNN}$ & 65.3 & 55.0 & 52.4 & 48.3 & 18.2 & 66.4 & 77.8 & 35.6 & 26.5 & 67.0 & 46.9 & 48.4 & 70.5 & 69.1 & 35.2 & 35.2 & 69.6 & 43.4 & 64.6 & 43.7 & 52.0 \\
\hline
\end{tabular}

TABLE 4

Evaluation of Window Refinement on the VOC 2007 Dataset, in Terms of Test-Set Average Precision (AP)

\begin{tabular}{|c|c|c|c|c|c|c|c|c|c|c|c|c|c|c|c|c|c|c|c|c|c|}
\hline & aero & bicy & bird & boa & bot & bus & car & cat & cha & cow & dtab & dog & hors & mbik & pers & plnt & she & sofa & trai & tv & Av. \\
\hline FV & 35.8 & 40.6 & 8.1 & 7.6 & 3.1 & 35.9 & 41.8 & 16.8 & 1.4 & 23.0 & 4.9 & 14.1 & 31.9 & 41.9 & 19.3 & 11.1 & 27.6 & 12.1 & 31.0 & 40.6 & 22.4 \\
\hline $\mathrm{CNN}$ & 32.1 & 46.9 & 28.4 & 12.0 & 9.6 & 39.4 & 45.5 & 16.2 & 14.8 & 33.1 & 11.6 & 14.0 & 31.2 & 39.3 & 13.1 & 19.7 & 30.5 & 23.4 & 37.0 & 19.6 & 25.9 \\
\hline $\mathrm{FV}+\mathrm{CNN}$ & 38.1 & 47.6 & 28.2 & 13.9 & 13.2 & 45.2 & 48.0 & 19.3 & 17.1 & 27.7 & 17.3 & 19.0 & 30.1 & 45.4 & 13.5 & 17.0 & 28.8 & 24.8 & 38.2 & 15.0 & 27.4 \\
\hline \multicolumn{22}{|c|}{ after window refinement } \\
\hline FV & 36.9 & 38.3 & 11.5 & 11.1 & 1.0 & 39.8 & 45.7 & 16.5 & 1.2 & 26.4 & 4.3 & 17.7 & 31.8 & 44.0 & 13.1 & 11.0 & 31.4 & 9.7 & 38.5 & 36.9 & 23.3 \\
\hline CNN & 40.4 & 43.5 & 29.5 & 11.4 & 9.4 & 42.2 & 47.3 & 25.6 & 7.6 & 33.8 & 15.8 & 27.7 & 37.4 & 46.4 & 20.5 & 19.9 & 30.2 & 23.5 & 40.6 & 19.6 & 28.6 \\
\hline $\mathrm{FV}+\mathrm{CNN}$ & 39.3 & 43.0 & 28.8 & 20.4 & 8.0 & 45.5 & 47.9 & 22.1 & 8.4 & 33.5 & 23.6 & 29.2 & 38.5 & 47.9 & 20.3 & 20.0 & 35.8 & 30.8 & 41.0 & 20.1 & 30.2 \\
\hline
\end{tabular}

TABLE 5

Comparison of WSL Detectors on PASCAL VOC 2007 in Terms of Test-Set Detection AP

\begin{tabular}{|c|c|c|c|c|c|c|c|c|c|c|c|c|c|c|c|c|c|c|c|c|c|}
\hline & ero & bicy & bird & boa & bot & bus & car & & cha & OW & tab & $\operatorname{dog}$ & lors & nbik & pers & plnt & she & sofa & trai & tv & Av. \\
\hline Pandey and Lazebnik'11 [35] & 11.5 & - & - & 3.0 & - & - & - & - & - & - & - & - & 20.3 & 9.1 & - & - & - & - & 13.2 & - & - \\
\hline Siva and Xiang'11 [45] & 13.4 & 44.0 & 3.1 & 3.1 & 0.0 & 31.2 & 43.9 & 7.1 & 0.1 & 9.3 & 9.9 & 1.5 & 29.4 & 38.3 & 4.6 & 0.1 & 0.4 & 3.8 & 34.2 & 0.0 & 13.9 \\
\hline Russakovsky et al.'12 [38] & 30.8 & 25.0 & - & 3.6 & - & 26.0 & - & - & - & - & - & - & 21.3 & 29.9 & - & - & - & - & - & - & 15.0 \\
\hline \multirow[t]{2}{*}{ Ours (FV-only) } & 36.9 & 38.3 & 11.5 & 11.1 & 1.0 & 39.8 & 45.7 & 16.5 & 1.2 & 26.4 & 4.3 & 17.7 & 31.8 & 44.0 & 13.1 & 11.0 & 31.4 & 9.7 & 38.5 & 36.9 & 23.3 \\
\hline & \multicolumn{21}{|c|}{ methods using additional training data } \\
\hline Song et al.'14 [46] & 27.6 & 41.9 & 19.7 & 9.1 & 10.4 & 35.8 & 39.1 & 33.6 & 0.6 & 20.9 & 10.0 & 27.7 & 29.4 & 39.2 & 9.1 & 19.3 & 20.5 & 17.1 & 35.6 & 7.1 & 22.7 \\
\hline Song et al.'14 [47] & 36.3 & 47.6 & 23.3 & 12.3 & 11.1 & 36.0 & 46.6 & 25.4 & 0.7 & 23.5 & 12.5 & 23.5 & 27.9 & 40.9 & 14.8 & 19.2 & 24.2 & 17.1 & 37.7 & 11.6 & 24.6 \\
\hline Bilen et al.'14 [8] & 42.2 & 43.9 & 23.1 & 9.2 & 12.5 & 44.9 & 45.1 & 24.9 & 8.3 & 24.0 & 13.9 & 18.6 & 31.6 & 43.6 & 7.6 & 20.9 & 26.6 & 20.6 & 35.9 & 29.6 & 26.4 \\
\hline Wang et al.'14 [53] & 48.8 & 41.0 & 23.6 & 12.1 & 11.1 & 42.7 & 40.9 & 35.5 & 11.1 & 36.6 & 18.4 & 35.3 & 34.8 & 51.3 & 17.2 & 17.4 & 26.8 & 32.8 & 35.1 & 45.6 & 30.9 \\
\hline Wang et al.'14 [53] +context & 48.9 & 42.3 & 26.1 & 11.3 & 11.9 & 41.3 & 40.9 & 34.7 & 10.8 & 34.7 & 18.8 & 34.4 & 35.4 & 52.7 & 19.1 & 17.4 & 35.9 & 33.3 & 34.8 & 46.5 & 31.6 \\
\hline Ours & 39.3 & 43.0 & 28.8 & 20.4 & 8.0 & 45.5 & 47.9 & 22.1 & 8.4 & 33.5 & 23.6 & 29.2 & 38.5 & 47.9 & 20.3 & 20.0 & 35.8 & 30.8 & 41.0 & 20.1 & 30.2 \\
\hline
\end{tabular}

Results for Pandey and Lazebnik [35] are taken from [37].

parts of the tables show the results for multi-fold MIL without window refinement, therefore, contain copies of the corresponding rows from Tables 1 and 2 . The bottom parts show the results for the window refinement method for the $\mathrm{FV}, \mathrm{CNN}$ and the combined features. We observe that the refinement method significantly improves the average CorLoc and AP scores for all three window descriptor types. In the case of $\mathrm{FV}+\mathrm{CNN}$ features, applying the window refinement method improves CorLoc and detection AP in 16 out of 20 classes, where we measure the largest three improvements in CorLoc for the classes horse, dog and cat. The instances of these three classes have deformable shapes, therefore, the weakly supervised localization tends to result in imprecise localizations or part localizations, some of which are corrected by the window refinement method. The four classes for which the window refinement method deteriorates CorLoc are bicycle, bottle, chair and potted-plant. These classes typically have highly textured and/or small instances, where the edge-driven objectness measure can be misleading. Finally, we note that the results obtained with refinement also include the addition of horizontal flips of the positive training windows. This has only a minor effect on performance: without these the detection mAP for the $\mathrm{FV}+\mathrm{CNN}$ features drops by only 0.4 to 29.8 percent. Overall, these results show that the FV and CNN features are complimentary, and that window refinement can improve localization performance.

\subsection{Comparison to State-of-the-Art WSL Detection}

We compare our multi-fold MIL approach to the state-ofthe-art in terms of detection AP in Table 5. We separate the recent work into two groups in terms of their utilization of auxiliary training data. To the best our knowledge, only three previous studies that do not use auxiliary training data reported detection AP scores on PASCAL VOC 2007. Other work, such as, e.g., that of Deselaers et al. [17], was evaluated only under simplified conditions, such as using viewpoint information and using images from a limited 
TABLE 6

Performance in Test-Set Detection mAP on VOC 2007 Using FV, CNN and FV+CNN Features, with Varying Degrees of Supervision

\begin{tabular}{lccccc}
\hline Supervision & Neg on Pos & Positive Set & FV & CNN & FV+CNN \\
\hline Image labels only & No & Non-diff/trunc & 22.4 & 25.9 & 27.4 \\
Cand box for one obj & No & Non-diff/trunc & 30.8 & 36.5 & 40.5 \\
Cand box for all obj & No & Non-diff/trunc & 30.7 & 35.7 & 38.4 \\
Cand box for all obj & Yes & Non-diff/trunc & 32.0 & 41.2 & 43.7 \\
Exact box for all obj & Yes & Non-diff/trunc & 32.8 & 40.5 & 43.6 \\
Exact box for all obj & Yes & All & 35.4 & 42.8 & 46.2 \\
\hline
\end{tabular}

number of classes. Russakovsky et al. [38] report mAP over all 20 classes, but report separate AP values for only six classes. Multi-fold MIL over the FV-only features with window refinement, results in a detection mAP of 23.3 percent, which is significantly better than the 13.9 and 15.0 percent reported in [45] and [38].

The second half of Table 5 presents the recent work that uses CNN-based features, which involves representation learning on the ImageNet dataset. For comparison, we use our multi-fold MIL approach over the FV+CNN features with window refinement. Our detection mAP of 30.2 percent is significantly better than the 22.7 and 24.6 percent by Song et al. [46], [47], and the 26.4 percent by Bilen et al. [8]. Wang et al. [53] report a detection $\mathrm{mAP}$ of 30.9 percent, and additionally an improved mAP of 31.6 percent mAP using the contextual rescoring method of [21]. Our detection mAP is comparable to Wang et al. [53] without inter-class context, and we obtain better AP scores in 11 out of 20 classes.

\subsection{Analysis of Performance and Failure Cases}

To analyze the causes of difficulty of WSL for object detection, we consider the performance of our detector when used in a fully supervised training setting. For the sake of brevity, we analyze the WSL results without applying window refinement.

There are several factors that change between the WSL and fully supervised training. In order to evaluate the importance of each factor, we progressively move from the original WSL setting to the fully supervised setting. In Table 6, we report the resulting mAP values for each step using the FV-only, CNN-only and FV+CNN features in the final three columns, respectively.

In WSL we have to determine the object locations in the positive training images. If in each positive training image we fix the object hypothesis to the candidate window that best overlaps with one of the ground-truth objects, we no longer need to use MIL training. In this case, we increase the detection mAP by 13.1 points to 40.5 w.r.t. the weakly supervised setting; see first and second row of Table 6. Even though this is a significant improvement w.r.t. WSL, there is still a gap of 5.7 percent in detection mAP compared to the fully supervised setting.

The remaining difference in performance is due to several factors, we list them now and give the performance improvements when making the WSL training scenario progressively more similar to the supervised one. (i) In WSL only one instance per positive training image is used. Including all instances instead makes a relatively minor
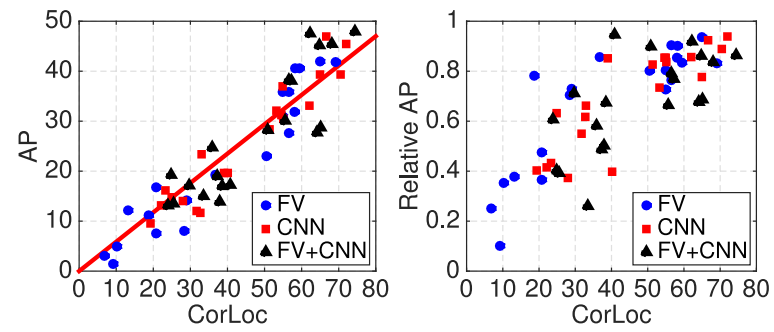

Fig. 7. AP versus CorLoc for multi-fold MIL (left), and ratio of WSL over supervised AP as a function of CorLoc (right) using the FV (blue circles), $\mathrm{CNN}$ (red squares) and FV+CNN (black triangles) representations. CorLoc and AP are measured on the training and test images, respectively. The left plot shows the line with least squares error for the data points.

effect on the performance, see the third row in Table 6 . (ii) In WSL hard-negative mining is based on negative images only, when positive images are used too performance rises to 43.7 percent $\mathrm{mAP}$ for the $\mathrm{FV}+\mathrm{CNN}$ features, as shown in the fourth row. (iii) WSL is based on the candidate windows, using the ground-truth windows instead makes a relatively small impact, see the fifth row. (iv) Finally, in WSL, we do not use positive training images marked as difficult or truncated, if these are added performance rises to 46.2 percent $\mathrm{mAP}$ for $\mathrm{FV}+\mathrm{CNN}$ features. ${ }^{2}$

These results show that the most important two factors are the use of correct training windows and hard-negative mining on positive training images. We also observe that multifold MIL achieves 59 percent of the representational performance limit (27.4 percent out of 46.2 percent mAP). With respect to the 40.5 percent $\mathrm{mAP}$ for training from ideal localizations, multi-fold MIL approach attains 68 percent of the WSL performance limit. Standard MIL (22.0 percent mAP, cf Table 2) attains only 54 percent of this performance limit.

In Fig. 7 we further analyze the results of our weakly supervised detector, and its relation to the optimally localized version. In the left panel, we visualize the close relationship between the per-class CorLoc and AP values for our multi-fold MIL detector. The three classes with lowest CorLoc are bottle, chair, and dining table using FVs, bottle, chair, and cat using CNNs, and bottle, cat, and person using the FV $+\mathrm{CNN}$ combination. Most instances of these classes appear in highly cluttered indoor images, and are often occluded by objects (dining table, chair), or have extremely variable appearance due to transparency (bottle) and deformation (cat, person). In the right panel, we plot the ratio between our WSL detection AP and the AP obtained with the same detector trained with optimal localization (the second row in Table 6). In this case there is also a clear relation with our CorLoc values. The relation is quite different, however, below and above 50 percent CorLoc. Below this threshold, due to the amount of noisy training examples, WSL tends to break down. Above this threshold, however, the training is able to cope with the noisy positive training examples, and the weakly supervised detector performs relatively well: on average above 80 percent relative to optimal localization.

In order to better understand the localization errors, we categorize each of our object hypotheses in the positive training images into one of the following five cases:

2. Note that our CNN-only fully-supervised mAP of 42.8 percent is comparable to the 44.7 percent of Girshick et al. [22], which uses similar CNN features. 


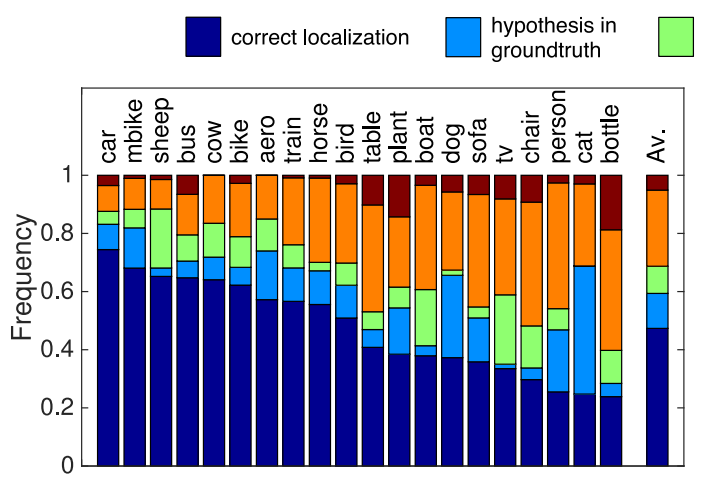

(a) multi-fold MIL groundtruth
in hypothesis $\square$ low overlap $\square$ no overlap

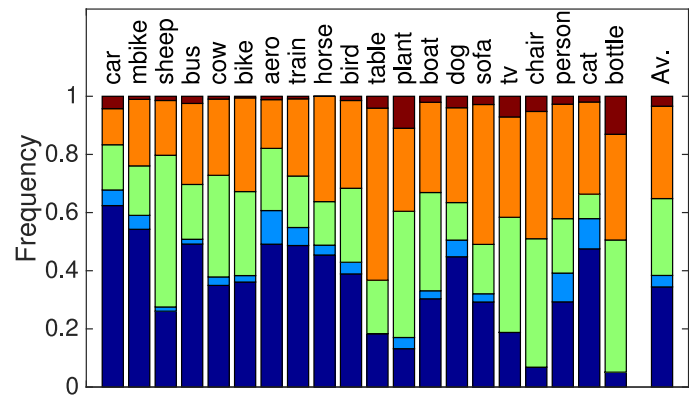

(b) standard MIL

Fig. 8. Per-class frequency of error modes, and averaged across all classes using FV+CNN features with 10-fold MIL and standard MIL training.

(i) correct localization (overlap $\geq 50$ percent), (ii) hypothesis completely inside ground-truth, (iii) reversed inclusion, (iv) none of the above, but non-zero overlap, and (v) no overlap. For the sake of brevity, we analyze only the WSL outputs for the FV+CNN features. In Fig. 8a we show the frequency of these five cases for each object category and averaged over all classes for multi-fold MIL. We observe that hypothesis in ground-truth category is the second largest error mode. For example, as expected from Fig. 4, most localization hypotheses for the class cat, and similarly for the class dog, are fully contained within a ground-truth window. Although the instances of this mis-localization category may significantly degrade CorLoc and AP measures, they could as well be interpreted as correct localizations in certain applications where it is not necessary to localize with bounding boxes fully covering target objects. Interestingly, we observe that, with 5.1 percent on average, the "no overlap" case is rare. This means that 94.9 percent of our object hypotheses overlap to some extent with a ground-truth object. This explains the fact that detector performance is relatively resilient to frequent mis-localization in the sense of the CorLoc measure.

Fig. $8 \mathrm{~b}$ presents the error distribution corresponding to the standard MIL training. Whereas hypothesis in groundtruth is more frequent than ground-truth in hypothesis for multi-fold MIL training, the situation is reversed for standard MIL training. This is a result of the fact that whereas multi-fold MIL is able localize most discriminative subregions of the object categories, standard MIL tends to get stuck after the first few iterations, resulting in too large bounding box estimates. The effect of multi-fold training on the distribution of different localization error types is similar when using the FV or CNN features alone.

Finally, we note that while multi-fold MIL using $k$ folds results in training $k$ additional classifiers per iteration, training duration grows sublinearly with $k$ since the number of re-localizations and hard-negative mining work does not change. In a single iteration of our implementation using FV features, (a) all SVM optimizations take 10.5 minutes for standard MIL and 42 minutes for 10-fold MIL, (b) re-localization on positive images take 5 minutes in both cases and (c) hard-negative mining takes 20 minutes in both cases. In total, standard MIL takes 35.5 minutes per iteration and 10-fold MIL takes 67 minutes per iteration, for a single class.

\subsection{Training with Mixed Supervision}

In our experiments so far, we have considered the WSL and fully supervised scenarios, where each training image is annotated with either class labels (WSL) or object bounding boxes (fully supervised). We now consider training using a mixture of the two paradigms, which we refer to as mixed supervision.

One way to combine weakly supervised and fully supervised training for object localization is to leverage an existing dataset of fully supervised training images of non-target classes during WSL of a new object category detector, also referred to as transfer learning, see, e.g., [17], [41]. Such an approach, however, does not provide any fully supervised example for the target class and does not allow hard negative mining on the positive images, both of which are important factors as shown in our previous analysis.

We, instead, consider a setup where a subset of the positive training images for each class is fully supervised. For this purpose, we randomly sample a subset of the positive training images and add ground-truth box annotations for all objects in them. These images are then excluded from the re-localization steps in the multi-fold training procedure and instead their ground-truth windows are used as positive training examples. We also use the fully supervised positive images for hard-negative mining, in addition to the negative images.

Fig. 9 presents detection AP scores as a function of the percentage of fully supervised positive training images. Each curve is obtained by evaluating the performance when the ratio of fully supervised images per class is set to values in $\{2.5,5,10,25,50,100\}$ percent. We also evaluate the baseline detection results where only the fully supervised images are used for training. We repeat each experiment twice and average the AP scores. In each plot, the resulting mixed supervision and baseline curves are shown using solid and dotted lines, respectively. The horizontal axes are in logarithmic scale.

The leftmost panel in Fig. 9 shows the mixed supervision evaluation results for the classes bus, horse, train, and sheep, which we select for their similarity in performance to the average case for FVs (the latter is shown in the third panel). For these four classes, and on average, we observe a significant performance gain using mixed supervision compared to conventional full supervision.

The only two classes where mixed supervision is not more effective than fully supervised training for FVs are 

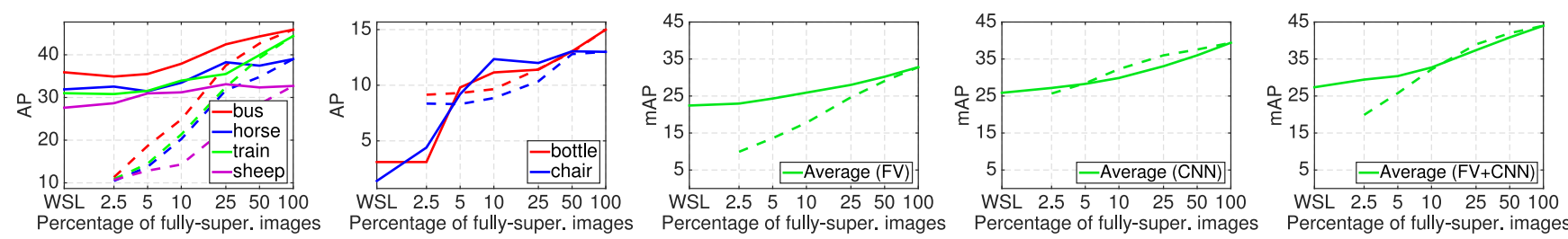

Fig. 9. Object detection results for training with mixed supervision. Each curve shows the test set detection AP as a function of the percentage of fully supervised positive training images. The horizontal axes are in logarithmic scale. The first two plots show per-class curves for selected classes using only FVs. The last three plots show the detection AP values averaged over all classes for the FV, CNN and FV+CNN features, respectively. The solid curves correspond to mixed supervision. The dotted curves correspond to results obtained by using only the fully-supervised examples.

bottle and chair, for which AP curves are presented in the second panel of Fig. 9. We note that bottle and chair are also the classes with the lowest CorLoc scores for multi-fold training, which explains why mixed supervised training does not work well in these cases.

In the third panel we observe that the fewer images are fully supervised, the more significant the benefit of additional weakly labeled images using FVs. Overall, we observe that the benefit of combining fully supervised images with weakly supervised ones is particularly significant when the ratio of fully supervised images is up to 50 percent for FV features.

The fourth panel in Fig. 9 presents the results for the $\mathrm{CNN}$ descriptors. We observe that training with mixed supervision improves the detection mAP compared to training with only the fully supervised examples when up to 5 percent of the positive training images are fully supervised. At larger fully supervised image percentages, training over only the fully supervised images outperforms mixed supervision based training. Regarding this result, we can interpret the $\mathrm{CNN}$ features as the outputs from a pre-trained classifier, and therefore, having a few training images can be sufficient for effectively learning a detection model over the $\mathrm{CNN}$ features. As a result, utilizing weakly supervised examples during training can sometimes deteriorate the detection performance due to the imperfect localizations provided by the WSL methods.

Finally, the rightmost panel in Fig. 9 presents the results for the $\mathrm{FV}+\mathrm{CNN}$ combination. We observe that training with mixed supervision is significantly beneficial when the ratio of fully supervised examples is up to 10 percent. Above this threshold, the performance of training with fully supervised examples is slightly better, similar to the CNN-only case.
Overall, the results suggest that fully supervised images can be successfully integrated into multi-fold WSL training in order to improve detection rates by annotating objects only in a small number of images. This holds in particular, when auxiliary training data, such as the ImageNet images used for training the CNN model, is not available. One possible direction for future work is to give more weight to fully supervised examples than to weakly supervised ones during classifier training, especially in the early MIL iterations.

\subsection{VOC 2010 Evaluation}

We now present an evaluation on the VOC 2010 dataset in order to verify the effectiveness of multi-fold training and the window refinement method on a second dataset. We are the first to present weakly supervised results on this dataset, and can therefore not compare to other weakly supervised methods. We show the resulting CorLoc values in Table 7 and detection AP results in Table 8. Overall, our results on VOC 2010 are similar to those on the 2007 dataset in the sense that multi-fold MIL significantly improves the WSL performance compared to standard MIL training, especially when high-dimensional FV descriptors are included. Using multi-fold MIL over the combined FV and CNN features results in 24.7 percent $\mathrm{mAP}$, which is significantly better than 21.9 percent $\mathrm{mAP}$ by standard MIL. The window refinement method further improves multi-fold MIL performance from 24.7 to 27.4 percent $\mathrm{mAP}$.

If we train the object detectors in a fully supervised manner, we obtain 33.6 percent $\mathrm{mAP}$ using the FV features, and 37.7 percent $\mathrm{mAP}$ using the $\mathrm{CNN}$ features. This verifies that we have an effective object representation outperforming DPMs [23] (29.6 percent mAP). On this dataset, the highest fully supervised detection result without using auxiliary data is 39.7 percent mAP [55]. We note that whereas the

TABLE 7

Comparison of Standard MIL Training versus Our 10-fold MIL on VOC 2010 in Terms of Training Set Localization Accuracy (CorLoc) aero bicy bird boa bot bus car cat cha cow dtab dog hors mbik pers plnt she sofa trai tv Av. standard MIL

FV

CNN

$\mathrm{FV}+\mathrm{CNN}$

$\mathrm{FV}+\mathrm{CNN}+$ Refinement $\begin{array}{llllllllllllll}58.9 & 45.2 & 33.7 & 24.1 & 6.7 & 66.1 & 43.3 & \mathbf{5 0 . 6} & 16.2 & 36.0 & 25.5 & 41.8 & 53.4 & 57.5\end{array}$

$\begin{array}{llllllllllllll}54.8 & 60.1 & 52.3 & 40.2 & 26.6 & 73.9 & 64.1 & 23.4 & 35.7 & 58.1 & 24.5 & 32.4 & 71.3 & 63.8\end{array}$

$\begin{array}{lllllllllllll}60.2 & 53.9 & 48.5 & 34.2 & 12.6 & 71.0 & 52.6 & 44.1 & 23.3 & 37.2 & 25.5 & 45.3 & 60.2\end{array}$

$\begin{array}{lllllllllllll}\mathbf{6 2 . 7} & 56.3 & 52.8 & 39.6 & 13.5 & 71.4 & 58.7 & 47.3 & 23.9 & 44.8 & 27.7 & \mathbf{5 4 . 4} & 65.9\end{array}$ $\begin{array}{llllllll}63.8 & 28.0 & 36.4 & 61.6 & 44.7 & 48.1 & 55.3 & 47.8\end{array}$

$\begin{array}{llllllll}61.3 & 36.0 & 15.3 & 36.6 & 34.0 & 51.0 & 31.8 & 41.7\end{array}$

$\begin{array}{llllllll}66.7 & 38.0 & 19.0 & 46.8 & 34.0 & 57.8 & 38.7 & 46.0\end{array}$

multi-fold MIL

\begin{tabular}{|c|c|c|c|c|c|c|c|c|c|c|c|c|c|c|c|c|c|c|c|c|c|}
\hline & 7.3 & 7.1 & 6.2 & 34.8 & 24.9 & 68.9 & 59.8 & 18.9 & 1.3 & 52.9 & 26.6 & 32.2 & 44.1 & 60.7 & 33.7 & 17.3 & 63.9 & 32.6 & 48.1 & 66.6 & \\
\hline NN & & 9.1 & 52.6 & & 7.1 & & & & & & & & & & & & & & & & \\
\hline & & 6 & 53. & 38. & D & 77.7 & & & & & & & & & & & & & & 49.7 & \\
\hline $\mathrm{V}+\mathrm{CNN}+$ Refinement & 1.1 & 65.0 & 59.2 & 44.3 & 28.3 & 80.6 & 69.7 & 31.2 & 42.8 & 73.3 & 38.3 & 50.2 & 74.9 & 70.9 & 37.3 & 37.1 & 65.3 & 55.3 & 61.7 & 58.2 & 55 \\
\hline
\end{tabular}


TABLE 8

Comparison of Standard MIL Training versus Our 10-fold MIL on VOC 2010 in Terms of Test set AP Measure

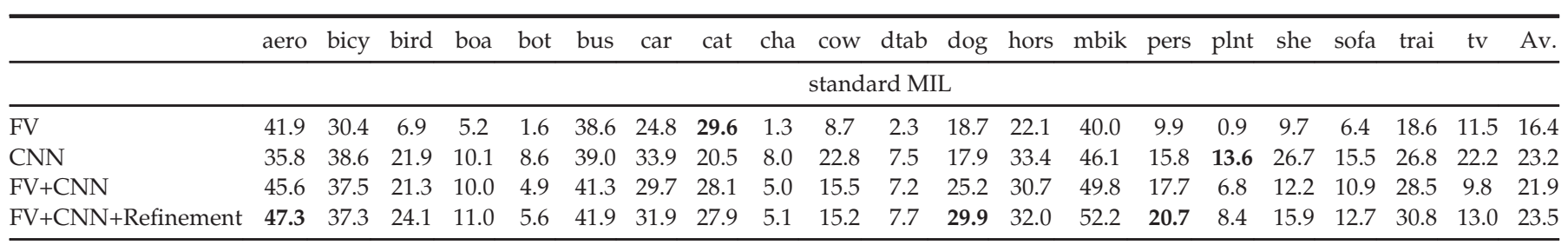

multi-fold MIL

\begin{tabular}{|c|c|c|c|c|c|c|c|c|c|c|c|c|c|c|c|c|c|c|c|c|c|}
\hline FV & 27.9 & 23.2 & 8.1 & 11.8 & 9.6 & 35.7 & 31.3 & 10.7 & 3.6 & 14.9 & 6.0 & 12.8 & 18.6 & 41.8 & 16.3 & 3.0 & 27.6 & 10.3 & 22.4 & 34.6 & 18.5 \\
\hline $\mathrm{CNN}$ & 34.7 & 39.1 & 21.9 & 10.5 & 8.8 & 37.7 & 34.4 & 18.1 & 10.1 & 26.4 & 11.2 & 16.5 & 33.0 & 44.7 & 15.6 & 13.2 & 26.2 & 15.6 & 24.8 & 24.8 & 23.4 \\
\hline $\mathrm{FV}+\mathrm{CNN}$ & 42.2 & 41.5 & 22.5 & 11.3 & 8.6 & 41.7 & 36.1 & 19.4 & 13.3 & 24.3 & 14.5 & 21.3 & 32.7 & 48.3 & 15.2 & 11.3 & 25.0 & 18.0 & 27.9 & 18.4 & 24.7 \\
\hline $\mathrm{FV}+\mathrm{CNN}+$ Refinement & 44.6 & 42.3 & 25.5 & 14.1 & 11.0 & 44.1 & 36.3 & 23.2 & 12.2 & 26.1 & 14.0 & 29.2 & 36.0 & 54.3 & 20.7 & 12.4 & 26.5 & 20.3 & 31.2 & 23.7 & 27.4 \\
\hline
\end{tabular}

CNN model we use is trained on the ImageNet images only, Girshick et al. [22] utilize a CNN model fine-tuned on the VOC ground-truth boxes, which leads to a better fullysupervised detection performance of 53.7 percent mAP. We plan to explore weakly supervised CNN fine-tuning in future work.

\section{CONCLUSIONS}

In this article, we have introduced a multi-fold multiple instance learning approach for weakly supervised object detection, which avoids the degenerate localization performance observed without it. Second, we have presented a contrastive background descriptor, which encourages the detection model to learn the differences between the objects and their context. Third, we have designed a window refinement method, which improves the localization accuracy by using an edge-driven objectness prior.

We have evaluated our approach and compared it to state-of-the-art methods using the VOC 2007 dataset. Our results show that multi-fold MIL effectively handles highdimensional descriptors, which allows us to obtain state-ofthe-art results by jointly using FV and CNN features. On the VOC 2010 dataset we observe similar improvements by using our multi-fold MIL method.

A detailed analysis of our results shows that, in terms of test set detection performance, multi-fold MIL attains 68 percent of the MIL performance upper-bound, which we measure by selecting one correct training example from each positive image, for the combined FV and CNN features.

\section{ACKNOWLEDGMENTS}

This work was supported by the European integrated project AXES and the ERC advanced grant ALLEGRO. Most of this work was done when R. G. Cinbis was with LEAR team, Inria Grenoble Rhône-Alpes, Laboratoire Jean Kuntzmann, CNRS, University Grenoble Alpes, France.

\section{REFERENCES}

[1] B. Alexe, T. Deselaers, and V. Ferrari, "What is an object?" in Proc. IEEE Conf. Comput. Vis. Pattern Recog., 2010, pp. 73-80.

[2] B. Alexe, T. Deselaers, and V. Ferrari, "Measuring the objectness of image windows," IEEE Trans. Pattern Anal. Mach. Intell., vol. 34, no. 11, pp. 2189-2202, Nov. 2012.

[3] F. Alted, "Why modern CPUs are starving and what can be done about it," Comput. Sci. Eng., vol. 12, no. 2, pp. 68-71, 2010.
[4] S. Bagon, O. Brostovski, M. Galun, and M. Irani, "Detecting and sketching the common," in Proc. IEEE Conf. Comput. Vis. Pattern Recog., 2010, pp. 33-40.

[5] Y. Bengio, J. Louradour, R. Collobert, and J. Weston, "Curriculum learning," in Proc. 28th Annu. Int. Conf. Mach. Learn., 2009, pp. 41-48.

[6] T. Berg, A. Berg, J. Edwards, M. Maire, R. White, Y. Teh, E. Learned-Miller, and D. Forsyth, "Names and faces in the news," in Proc. IEEE Conf. Comput. Vis. Pattern Recog., 2004, pp. 848-854.

[7] H. Bilen, V. Namboodiri, and L. Van Gool, "Object and action classification with latent window parameters," Int. J. Comput. Vis., vol. 106, no. 3, pp. 237-251, 2014.

[8] H. Bilen, M. Pedersoli, and T. Tuytelaars, "Weakly supervised object detection with posterior regularization," in Proc. Brit. Mach. Vis. Conf., 2014.

[9] M. Blaschko, A. Vedaldi, and A. Zisserman, "Simultaneous object detection and ranking with weak supervision," in Proc. Adv. Neural Inf. Process. Syst., 2010, pp. 235-243.

[10] D. Blei, A. Ng, and M. Jordan, "Latent Dirichlet allocation," J. Mach. Learn., vol. 3, pp. 993-1022, 2003.

[11] M. Cho, S. Kwak, C. Schmid, and J. Ponce, "Unsupervised object discovery and localization in the wild: Part-based matching with bottom-up region proposals," in Proc. IEEE Conf. Comput. Vis. Pattern Recog., 2015, pp. 1201-1210.

[12] O. Chum and A. Zisserman, "An exemplar model for learning object classes," in Proc. IEEE Conf. Comput. Vis. Pattern Recog., 2007, pp. 1-8.

[13] R. Cinbis, J. Verbeek, and C. Schmid, "Segmentation driven object detection with Fisher vectors," in Proc. Int. Conf. Comput. Vis., 2013, pp. 2968-2975.

[14] R. Cinbis, J. Verbeek, and C. Schmid, "Multi-fold MIL training for weakly supervised object localization," in Proc. IEEE Conf. Comput. Vis. Pattern Recog., 2014, pp. 2409-2416.

[15] D. Crandall and D. Huttenlocher, "Weakly supervised learning of part-based spatial models for visual object recognition," in Proc. 9th Eur. Conf. Comput. Vis., 2006, pp. 16-29.

[16] G. Csurka, C. Dance, L. Fan, J. Willamowski, and C. Bray, "Visual categorization with bags of keypoints," in Proc. ECCV Int. Workshop Statist. Learn. Comput. Vis., 2004.

[17] T. Deselaers, B. Alexe, and V. Ferrari, "Weakly supervised localization and learning with generic knowledge," Int. J. Comput. Vis., vol. 100, no. 3, pp. 257-293, 2012.

[18] T. Dietterich, R. Lathrop, and T. Lozano-Pérez, "Solving the multiple instance problem with axis-parallel rectangles," Artif. Intell., vol. 89, no. 1-2, pp. 31-71, 1997.

[19] M. Everingham, J. Sivic, and A. Zisserman, "Taking the bite out of automatic naming of characters in TV video," Image Vis. Comput., vol. 27, no. 5, pp. 545-559, 2009.

[20] M. Everingham, L. van Gool, C. Williams, J. Winn, and A. Zisserman, "The PASCAL visual object classes (VOC) challenge," Int. J. Comput. Vis., vol. 88, no. 2, pp. 303-338, 2010.

[21] P. Felzenszwalb, R. Grishick, D. McAllester, and D. Ramanan, "Object detection with discriminatively trained part based models," IEEE Trans. Pattern Anal. Mach. Intell., vol. 32, no. 9, pp. 16271645, Sep. 2010.

[22] R. Girshick, J. Donahue, T. Darrell, and J. Malik, "Rich feature hierarchies for accurate object detection and semantic segmentation," in Proc. IEEE Conf. Comput. Vis. Pattern Recog., 2013, pp. 580-587. 
[23] R. Girshick, P. Felzenszwalb, and D. McAllester. (2012). Discriminatively trained deformable part models, release 5 [Online]. Available: http:/ / people.cs.uchicago.edu/ rbg/latent-release5.

[24] C. Gu, P. Arbeláez, Y. Lin, K. Yu, and J. Malik, "Multi-component models for object detection," in Proc. Eur. Conf. Comput. Vis., 2012, pp. $445-458$.

[25] T. Hofmann, “Unsupervised learning by probabilistic latent semantic analysis," Mach. Learn., vol. 42, no. 1/2, pp. 177-196, 2001.

[26] H. Jégou, M. Douze, and C. Schmid, "Product quantization for nearest neighbor search," IEEE Trans. Pattern Anal. Mach. Intell., vol. 33, no. 1, pp. 117-128, Jan. 2011.

[27] Y. Jia, E. Shelhamer, J. Donahue, S. Karayev, J. Long, R. Girshick, S. Guadarrama, and T. Darrell, "Caffe: Convolutional architecture for fast feature embedding," in arXiv Preprint arXiv:1408.5093, 2014.

[28] G. Kim and A. Torralba, "Unsupervised detection of regions of interest using iterative link analysis," in Proc. Adv. Neural Inf. Process. Syst., 2009, pp. 4-2.

[29] A. Krizhevsky, I. Sutskever, and G. Hinton, "Imagenet classification with deep convolutional neural networks," in Proc. Adv. Neural Inf. Process. Syst., pp. 1106-1114, 2012.

[30] M. P. Kumar, B. Packer, and D. Koller, "Self-paced learning for latent variable models," in Proc. Adv. Neural Inf. Process. Syst., 2010, pp. 1189-1197.

[31] C. Lampert, M. Blaschko, and T. Hofmann, "Efficient subwindow search: A branch and bound framework for object localization," IEEE Trans. Pattern Analy. Mach. Intell., vol. 31, no. 12, pp. 21292142, Dec. 2009.

[32] S. Lazebnik, C. Schmid, and J. Ponce, "Beyond bags of features: Spatial pyramid matching for recognizing natural scene categories," in Proc. IEEE Conf. Comput. Vis. Pattern Recog., 2006, pp. 2169-2178.

[33] Y. Nesterov, "Smooth minimization of non-smooth functions," Math. Program., vol. 103, no. 1, pp. 127-152, 2005.

[34] M. Nguyen, L. Torresani, F. de la Torre, and C. Rother, "Weakly supervised discriminative localization and classification: A joint learning process," in Proc. Int. Conf. Comput. Vis., 2009, pp. 19251932.

[35] M. Pandey and S. Lazebnik, "Scene recognition and weakly supervised object localization with deformable part-based models," in Proc. Int. Conf. Comput. Vis., 2011, pp. 1307-1314.

[36] O. Parkhi, A. Vedaldi, C. Jawahar, and A. Zisserman, "The truth about cats and dogs," in Proc. Int. Conf. Comput. Vis., 2011, pp. 1427-1434.

[37] A. Prest, C. Leistner, J. Civera, C. Schmid, and V. Ferrari, "Learning object class detectors from weakly annotated video," Proc. IEEE Conf. Comput. Vis. Pattern Recog., 2012, pp. 3282-3289.

[38] O. Russakovsky, Y. Lin, K. Yu, and L. Fei-Fei, “Object-centric spatial pooling for image classification," in Proc. 12th Eur. Conf. Comput. Vis., 2012, pp. 1-15.

[39] J. Sánchez, F. Perronnin, T. Mensink, and J. Verbeek, "Image classification with the Fisher vector: Theory and practice," Int. J. Comput. Vis., vol. 105, no. 3, pp. 222-245, 2013.

[40] Z. Shi, T. Hospedales, and T. Xiang, "Bayesian joint topic modelling for weakly supervised object localisation," in Proc. Int. Conf. Comput. Vis., 2013, pp. 2984-2991.

[41] Z. Shi, P. Siva, T. Xiang, and Q. Mary, "Transfer learning by ranking for weakly supervised object annotation," in Proc. Brit. Mach. Vis. Conf., 2012.

[42] S. Singh, A. Gupta, and A. Efros, "Unsupervised discovery of mid-level discriminative patches," in Proc. 12th Eur. Conf. Comput. Vis., 2012, pp. 73-86.

[43] P. Siva, C. Russell, and T. Xiang, "In defence of negative mining for annotating weakly labelled data," in Proc. Eur. Conf. Comput. Vis., 2012, pp. 594-608.

[44] P. Siva, C. Russell, T. Xiang, and L. Agapito, "Looking beyond the image: Unsupervised learning for object saliency and detection," in Proc. IEEE Conf. Comput. Vis. Pattern Recog., 2013, pp. 3238-3245.

[45] P. Siva and T. Xiang, "Weakly supervised object detector learning with model drift detection," in Proc. Int. Conf. Comput. Vis., 2011, pp. 343-350.

[46] H. Song, R. Girshick, S. Jegelka, J. Mairal, Z. Harchaoui, and T. Darrell, "On learning to localize objects with minimal supervision," in Proc. Int. Conf. Mach. Learn., 2014, pp. 1611-1619.
[47] H. Song, Y. Lee, S. Jegelka, and T. Darrell, "Weakly-supervised discovery of visual pattern configurations," in Proc. Adv. Neural Inf. Process. Syst., 2014, pp. 1637-1645.

[48] Z. Song, Q. Chen, Z. Huang, Y. Hua, and S. Yan, "Contextualizing object detection and classification," in Proc. IEEE Conf. Comput. Vis. Pattern Recog., 2011, pp. 1585-1592.

[49] J. Uijlings, K. van de Sande, T. Gevers, and A. Smeulders, "Selective search for object recognition," Int. J. Comput. Vis., vol. 104, no. 2, pp. 154-171, 2013.

[50] K. van de Sande, C. Snoek, and A. Smeulders, "Fisher and VLAD with FLAIR," in Proc. IEEE Conf. Comput. Vis. Pattern Recog., 2014, pp. 2377-2384.

[51] J. Verbeek and B. Triggs, "Region classification with Markov field aspect models," in Proc. IEEE Conf. Comput. Vis. Pattern Recog., 2007, pp. 1-8.

[52] S. Vijayanarasimhan and K. Grauman, "Large-scale live active learning: Training object detectors with crawled data and crowds," in Proc. IEEE Conf. Comput. Vis. Pattern Recog., 2011, pp. $1449-1456$.

[53] C. Wang, W. Ren, K. Huang, and T. Tan, "Weakly supervised object localization with latent category learning," in Proc. 13th Eur. Conf. Comput. Vis., 2014, pp. 431-445.

[54] J. Wang, J. Yang, K. Yu, F. Lv, T. Huang, and Y. Gong, "Localityconstrained linear coding for image classification," in Proc. IEEE Conf. Comput. Vis. Pattern Recog., 2010, pp. 3360-3367.

[55] X. Wang, M. Yang, S. Zhu, and Y. Lin, "Regionlets for generic object detection," in Proc. Int. Conf. Comput. Vis., 2013, pp. 17-24.

[56] C. Zitnick and P. Dollár, "Edge boxes: Locating object proposals from edges," in Proc. Eur. Conf. Comput. Vis., 2014, pp. 391-405.

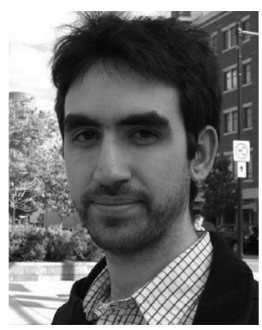

Ramazan Gokberk Cinbis graduated from Bilkent University, Turkey, in 2008, and received the MA degree from Boston University, in 2010. He was a doctoral student in the LEAR team, at INRIA Grenoble, from 2010 until 2014, and received the $\mathrm{PhD}$ degree in computer science from Université de Grenoble, France, in 2014. He is currently an assistant professor at the Department of Computer Engineering, Bilkent University, Ankara, Turkey. His research interests include computer vision and machine learning.

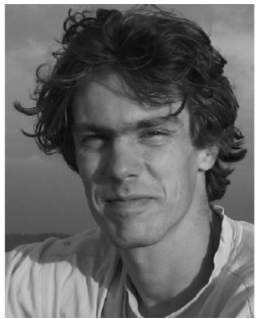

Jakob Verbeek received the $\mathrm{PhD}$ degree in computer science in 2004 from the University of Amsterdam, The Netherlands. After being a postdoctoral researcher at the University of Amsterdam and at INRIA Rhône-Alpes, he has been a full-time researcher at INRIA, Grenoble, France, since 2007. His research interests include machine learning and computer vision, with special interest in applications of statistical models in computer vision.

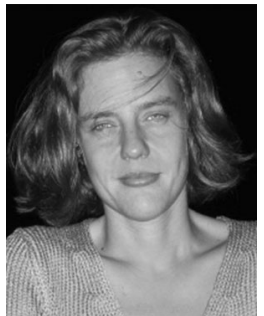

Cordelia Schmid received the MS degree in computer science from the University of Karlsruhe and the doctorate degree from the Institute National Polytechnique de Grenoble. She is a research director at INRIA Grenoble where she directs the LEAR team. In 2006 and 2014, she received the Longuet-Higgins prize for fundamental contributions in computer vision that have withstood the test of time. In 2012, she received an ERC advanced grant. She is a fellow of the IEEE.

$\triangleright$ For more information on this or any other computing topic, please visit our Digital Library at www.computer.org/publications/dlib. 